\title{
agNANO
}

\section{. Effect of Chiral Ligand Concentration and - Binding Mode on Chiroptical Activity of CdSe/ CdS Quantum Dots}

\author{
${ }_{4}$ Vera A. Kuznetsova, ${ }^{*}, \dagger, \downarrow, \nabla$ Eric Mates-Torres, ${ }^{\dagger, \nabla}$ Nadezda Prochukhan, ${ }^{\dagger}$ Madeline Marcastel, ${ }^{\dagger}$ \\ ${ }_{5}$ Finn Purcell-Milton, ${ }^{\dagger, \S(0)}$ John O’Brien, ${ }^{\dagger}$ Anastasia K. Visheratina, ${ }^{\ddagger}$ (i) Marina Martinez-Carmona, ${ }^{(1)}$ \\ 6 Yulia Gromova, ${ }^{\dagger}$ Max Garcia-Melchor, ${ }^{*}{ }^{\dagger}$ a ${ }^{\circ}$ and Yurii K. Gun'ko*, ${ }^{\dagger}, \S(0)$ \\ $7^{\dagger}$ School of Chemistry, CRANN and AMBER Research Centres, Trinity College Dublin, College Green, Dublin 2, Ireland \\ 8 ITMO University, St. Petersburg 197101, Russia \\ $9{ }^{\S}$ BEACON, Bioeconomy SFI Research Centre, University College Dublin, Dublin 4, Ireland
}

10 S Supporting Information

11 ABSTRACT: Chiroptically active fluorescent semiconductor nano12 crystals, quantum dots (QDs), are of high interest, from a 13 theoretical and technological point of view, because they are 14 promising candidates for a range of potential applications. Optical 15 activity can be induced in QDs by capping them with chiral 16 molecules, resulting in circular dichroism (CD) signals in the range 17 of the QD ultraviolet-visible (UV-vis) absorption. However, the 18 effects of the chiral ligand concentration and binding modes on the 19 chiroptical properties of QDs are still poorly understood. In the 20 present study, we report the strong influence of the concentration of 21 a chiral amino acid (cysteine) on its binding modes upon the 22 surface of $\mathrm{CdSe} / \mathrm{CdS} \mathrm{QDs}$, resulting in varying QD chiroptical 23 activity and corresponding CD signals. Importantly, we demon24 strate that the increase of cysteine concentration is accompanied by nanoscale and for the design of advanced optically active nanomaterials.

the growth of the QD CD intensity, reaching a certain critical point, after which it starts to decrease. The intensity of the CD signal varies by almost an order of magnitude across this range. Nuclear magnetic resonance and Fourier transform infrared data, supported by density functional theory calculations, reveal a change in the binding mode of cysteine molecules from tridentate to bidentate when going from low to high concentrations, which results in a change in the CD intensity. Hence, we conclude that the chiroptical properties of QDs are dependent on the concentration and binding modes of the capping chiral ligands. These findings are very important for understanding chiroptical phenomena at the

KEYWORDS: chirality, quantum dots, chiroptical activity, cysteine, ligand concentration, binding mode, density functional theory

33
34 hirality is among the most fascinating occurrences in the natural world. A chiral molecule is one that has two mirror-image forms, i.e., enantiomers, which are 36 nonsuperimposable in three dimensions. Well-known examples 37 of chiral molecules are proteins, DNA, sugars, amino acids, 38 enzymes, and drugs such as ibuprofen and L-Dopa, which is a 39 drug for the treatment of Parkinson's disease. Overall, chirality 40 is one of the most important factors in biomolecular 41 recognition, and, therefore, chiral compounds play a very 42 significant role in chemistry, biology, pharmacy and medicine. 43 Chirality has also been envisaged to play an important role in 44 nanotechnology, and recently, the area of chiral nanomaterials 45 has received a great deal of attention, because of the range of 46 potential applications offered by these materials. Chiroptically 47 active quantum dots (QDs)-fluorescent semiconductor nanocrystals with tunable optical properties-have also been 48 intensively studied in the last two decades ${ }^{1,2}$ since they were 49 first reported in 2007. ${ }^{3}$ Chiral QDs have several potential 50 applications, ${ }^{1,2,4-7}$ such as biological sensors, ${ }^{8-17}$ anticounter- 51 feiting material, ${ }^{18,19}$ as well as components in photonic ${ }^{20-23}{ }_{52}$ and in spin-polarized devices. ${ }^{24-26}$

Optical activity can be induced in QDs by functionalization 54 with chiral ligands via a relatively simple post-synthetic phase- 55 transfer ligand exchange. ${ }^{1,4,27-40}$ In this process, the initial 56 hydrophobic ligands in QDs produced by the hot injection 57

Received: September 23, 2019

Accepted: November 7, 2019

Published: November 7, 2019 
58 synthesis in organic medium are typically exchanged by chiral 59 hydrophilic ligands, accompanied by the transfer of QDs into 60 the aqueous phase. ${ }^{16,33,35,37}$ In this case, QDs exhibit optical 61 activity in their absorption region, unlike the chiral ligand 62 molecules, which typically absorb light only in the deep UV 63 region. $^{33,37}$ The origin of induced chirality can be explained by 64 the chiral distortion of QD surface atoms upon the adsorption 65 of chiral ligands, ${ }^{3,34,41,42}$ or the hybridization of the energy 66 levels of the QD and chiral ligand molecules leading to the 67 splitting of QD hole-electron levels into two sublevels with 68 different absorptions of circularly polarized light. ${ }^{33,38,40,43-47}$

69 The phenomenon of induced chiroptical activity in nano70 particles has attracted considerable attention, and, currently, it 71 is being intensively studied. The shape of QD circular 72 dichroism (CD) spectra and the magnitude of induced CD 73 bands are dependent on many factors, including the nature of 74 the material and the size of the QD core, ${ }^{29,40}$ QD shape and 75 anisotropy, ${ }^{1,31,35-37,48-50}$ the nature and thickness of the QD 76 shell, $^{33,34,40}$ and the chemical composition and coordination 77 modes of the ligands. ${ }^{27,28,32,51}$ The concentration of chiral 78 ligands used during the QD synthesis has also been reported to 79 influence the intensity of CD signal of nanoparticles, ${ }^{31,52}$ but 80 the addition of different amounts of ligands to the synthetic 81 medium leads to the formation of nanoparticles with different 82 morphology, which is proven to be the main reason for the 83 changes observed in the CD spectra. Hence, the effect of the 84 chiral ligand concentration is still unclear, and it is yet to be 85 demonstrated.

86 Herein we report a detailed investigation of the effect of 87 chiral L-cysteine ligands (hereafter referred simply as Cys) 88 concentration and binding mode on the CD intensity of the ${ }_{89} \mathrm{CdSe} / \mathrm{CdS} \mathrm{QDs}$, which were prepared by an exchange of 90 achiral organic ligands with chiral Cys ligands. We have found 91 that the dependence of $\mathrm{QD} \mathrm{CD}$ signal intensity on Cys 92 concentration is nonlinear; initially, the $\mathrm{CD}$ signal peak 93 intensity increases with Cys concentration, followed by a 94 decrease when a further excess of ligands is added. Moreover, 95 CD intensity changes dramatically, depending on ligand 96 concentration, by up to 1 order of magnitude, which makes 97 it one of the key factors that should be taken into account 98 during the investigation of chiroptical properties of nano99 particles. By using NMR and FTIR spectroscopy and density 100 functional theory (DFT) calculations, we have shown that, 101 while at low concentrations, Cys ligands are bound to the QD 102 surface in a tridentate coordination, an increase of the Cys 103 concentration switches the coordination trend toward 104 bidentate binding modes. Furthermore, our DFT approach 105 has allowed us to assess the stabilization of the Cys ligands 106 quantitatively via noncovalent interactions at different ligand 107 concentrations and to clearly pinpoint these interactions. We 108 believe these studies are of great theoretical and practical 109 importance, because they provide a fundamental under110 standing of chiroptical phenomena at the nanoscale, by 111 considering chiral ligand concentrations, ligand-ligand inter112 actions, and their binding modes at the QD surface. Hence, 113 this research is envisioned to contribute to the design of 114 advanced chiroptically active nanomaterials by leveraging an 115 optimal ligand concentration to maximize the $\mathrm{CD}$ response 116 and improve signal-to-noise ratio for potential applications 117 such as CD spectroscopy, optical sensing, metamaterials, and 118 nanophotonics.

\section{RESULTS AND DISCUSSION}

Effect of Cys Concentration on the Chiroptical 120 Activity of CdSe/CdS Core/Shell QDs. CdSe/CdS QDs 121 (hereafter referred as QDs) were synthesized via the well- 122 documented SILAR hot injection technique. ${ }^{33,53}$ QDs were 123 characterized by UV-Vis, PL, and CD spectroscopy and 124 transmission electron microscopy. The diameter of the QDs 125 obtained was $5.2 \pm 0.8 \mathrm{~nm}$, while the thickness of the CdS 126 shell was $1.2 \pm 0.4 \mathrm{~nm}$, which corresponds to five monolayers 127 of CdS. Full characterization of QDs can be found in the 128 Supporting Information. Original hydrophobic ligands (mostly 129 oleylamine) of QDs were subsequently displaced by L- and D- 130 cysteine, using a previously reported phase-transfer proce- 131 dure. ${ }^{14}$ Briefly, a Cys solution in methanol was added to the 132 QD chloroform solution, then shaken, and left for 2 min to 133 allow Cys to replace the hydrophobic ligands. After that, an 134 aqueous $0.01 \mathrm{M} \mathrm{KOH}$ solution was added and the Cys- 135 functionalized QDs were transferred to the aqueous phase. As 136 a result, the QDs became water-soluble and chiroptically 137 active, which is reflected in the appearance of mirror-imaged 138 $\mathrm{CD}$ signals in the region of QD absorbance including the 139 excitonic area (Figure 1). CD signal corresponding to the QD $140 \mathrm{fl}$

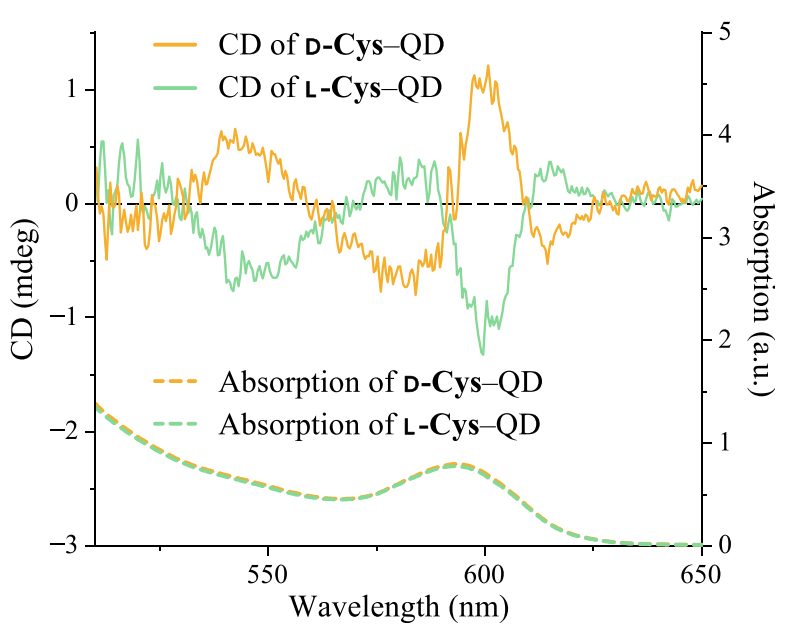

Figure 1. CD spectra (solid lines) and UV-vis spectra (dotted lines) of D-Cys-functionalized CdSe/CdS QDs (orange) and L-Cysfunctionalized $\mathrm{CdSe} / \mathrm{CdS}$ QDs (green). Cysteine concentration = $0.42 \mathrm{mg} / \mathrm{mL}$.

exciton is produced due to the hybridization of the 141 degenerated valence band energy level of QDs and HOMO 142 level of Cys molecules yielding two sublevels. The resulting 143 optical transitions from these sublevels have a contra- 144 directional rotary strength, which is reflected in the splitting 145 of CD signal for positive and negative bands crossing zero in 146 the vicinity of exciton maximum. ${ }^{27-29,33,34,40,49} 147$

To study the influence of Cys concentration on QD 148 chiroptical response intensity, a ligand exchange was 149 performed using different amounts of Cys, varying from 0.1150 $\mathrm{mg} / \mathrm{mL}$ to $1.6 \mathrm{mg} / \mathrm{mL}$. We note that it was not technically 151 possible to perform the procedure with higher amounts of Cys 152 ligand, because of the limited solubility of Cys in methanol. 153 Thus, extra amounts ( 3 and $10 \mathrm{mg} / \mathrm{mL}$ ) were added to the 154 aqueous solutions of Cys QDs to achieve the even-higher Cys 155 concentration of 4.6 and $11.6 \mathrm{mg} / \mathrm{mL}$. G-factor curves of Cys- 156 functionalized QDs after phase transfer with different amounts 157 of Cys are illustrated in Figure 2a. The $G$-factor was used $158 \mathrm{f} 2$ 
a)

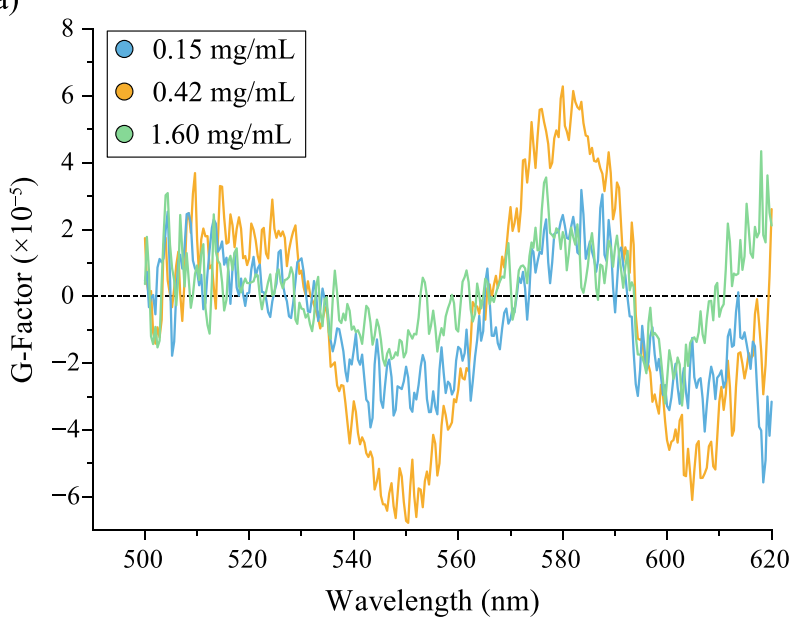

b)

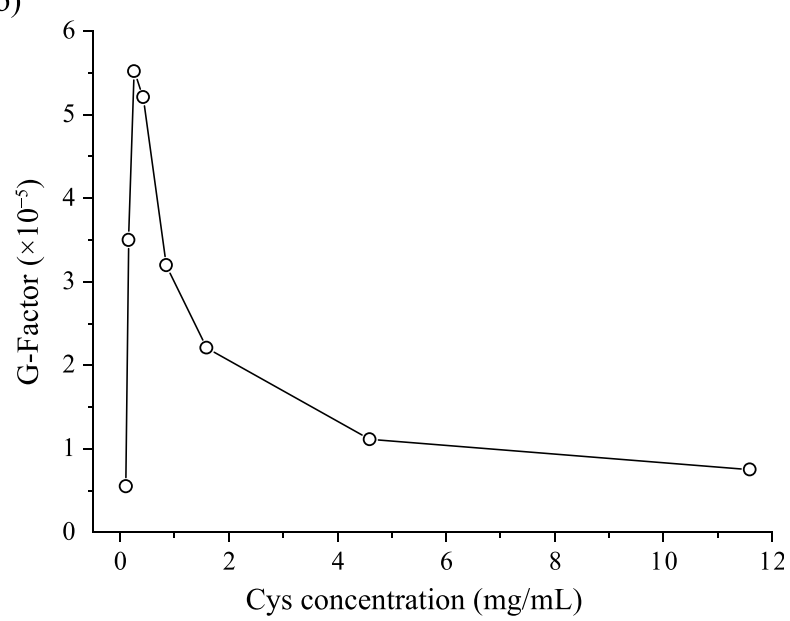

Figure 2. (a) $G$-factor graphs of Cys-functionalized CdSe/CdS QDs with varying Cys concentrations: 0.15 , 0.42 , and $1.60 \mathrm{mg} / \mathrm{mL}$. (b) Dependence of the $G$-factor intensity in the excitonic region of Cys CdSe/CdS QDs at $605 \mathrm{~nm}$ on Cys concentration.

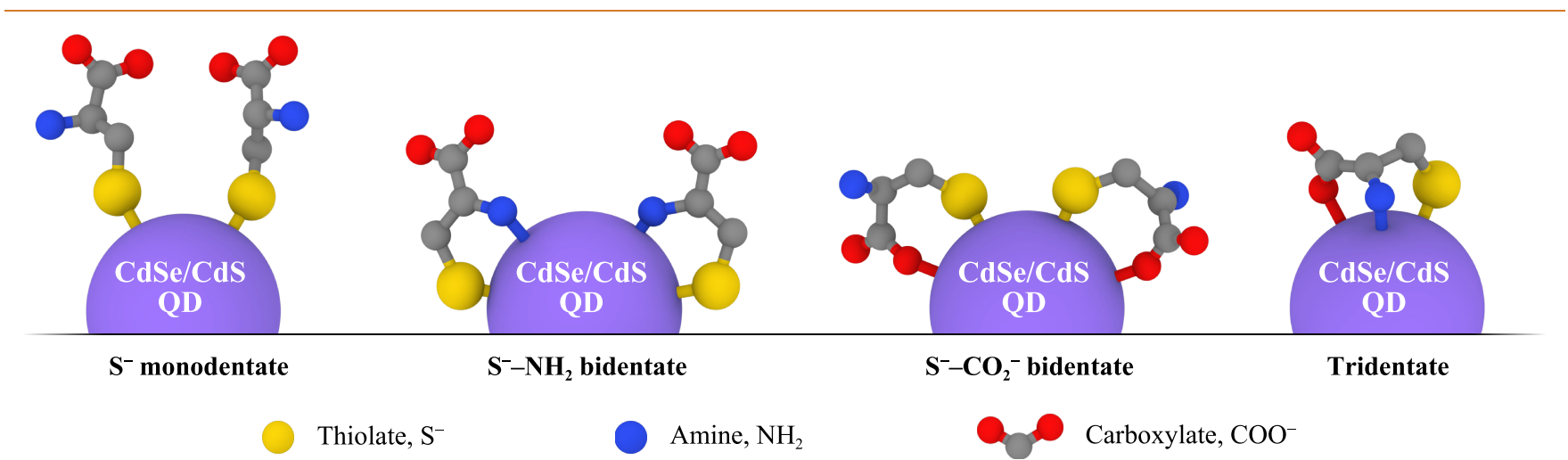

Figure 3. Possible binding modes of Cys ligand on the QD surface (from left to right): $\mathrm{S}^{-}$monodentate, $\mathrm{S}^{-}-\mathrm{NH}_{2}$ bidentate, $\mathrm{S}^{-}-\mathrm{CO}_{2}^{-}$ bidentate, and tridentate.

159 instead of $\mathrm{CD}$ signal to avoid the effect of the QD 160 concentration influence. The $G$-factor is defined as $G=\Delta \varepsilon$ / $161 \varepsilon=\left(A_{\mathrm{L}}-A_{\mathrm{R}}\right) / A$, where $A_{\mathrm{L}}$ and $A_{\mathrm{R}}$ are the absorbance of left162 handed and right-handed circularly polarized light, respec163 tively, and $A$ is the absorbance of unpolarized light. It was 164 found that the $G$-factor intensity varied with the Cys 165 concentration in a nonlinear fashion. Similar results were 166 also obtained for D-Cys QDs (see Figure S3 in the Supporting 167 Information). The dependence of the $G$-factor intensity of the 168 maximum peak corresponding to the $\mathrm{QD}$ excitonic region on 169 Cys concentration is shown in Figure $2 \mathrm{~b}$. The $G$-factor 170 intensity increased initially with Cys concentration. However, 171 after a critical Cys concentration (i.e., $0.26 \mathrm{mg} / \mathrm{mL}$ ) was 172 achieved, the QD $G$-factor intensity reached a maximum and 173 started to decrease. In light of these striking observations, we 174 decided to perform more-detailed investigations.

175 Determination of Cys Binding Mode on the QD 176 Surface. One explanation for the reduction of the $G$-factor 177 might be the change of the Cys binding mode with the $\mathrm{Cd}^{2+}$ 178 ions on the QD surface upon increasing the amount of Cys 179 ligands in solution. Indeed, it was previously reported that the 180 shape of the QD CD spectra is strongly dependent on the 181 coordination mode of chiral ligands on the QD sur182 face, ${ }^{27,28,32,54,55}$ but the dependence of the intensity of the 183 QD CD signals and the coordination of the ligands on their 184 concentration has not been explored to date.
The Cys ligand at the experimental $\mathrm{pH}$ of 13 has three 185 moieties: thiolate $\left(\mathrm{S}^{-}\right)$, carboxylate $\left(\mathrm{COO}^{-}\right)$, and amino 186 $\left(\mathrm{NH}_{2}\right)$ functional groups. Potentially, all of them can be 187 coordinated to $\mathrm{Cd}^{2+}$ ions on the $\mathrm{QD}$ surface, although $\mathrm{S}^{-}$has 188 the strongest affinity to $\mathrm{Cd}^{2+}$ ions. Thus, cysteine can bind to 189 the QD surface via all three groups (tridentate), via a 190 combination of $\mathrm{S}^{-}$and $\mathrm{NH}_{2}\left(\mathrm{~S}^{-}-\mathrm{NH}_{2}\right.$ bidentate) or $\mathrm{S}^{-}$and 191 $\mathrm{COO}^{-}\left(\mathrm{S}^{-}-\mathrm{COO}^{-}\right.$bidentate $)$, and solely via the $\mathrm{S}^{-} 192$ (monodentate). All of these possible Cys binding modes are 193 depicted in Figure 3.

Some investigations of Cys binding modes on the surface of 195 Cd-based clusters and nanoparticles have been previously 196 reported. For example, by performing multinuclear $\left({ }^{1} \mathrm{H},{ }^{13} \mathrm{C}, 197\right.$ ${ }^{77} \mathrm{Se},{ }^{15} \mathrm{~N},{ }^{113} \mathrm{Cd}$, and ${ }^{23} \mathrm{Na}$ ) solid-state $\mathrm{NMR}$ techniques, 198 Takegoshi et al. have shown that Cys ligand binds with $\mathrm{CdSe}_{34} 199$ magic clusters via coordination to $\mathrm{Cd}^{2+}$ ions as $\mathrm{S}^{-}-\mathrm{NH}_{2} 200$ bidentate and $\mathrm{S}^{-}$monodentate ligand, in amounts of $43 \% 201$ and $57 \%$, respectively. ${ }^{56-58}$ In another study using ${ }^{13} \mathrm{C}$ solid- 202 state $\mathrm{NMR}^{29}$ it was demonstrated that Cys binds to the 203 surface of $2.9 \mathrm{~nm} \mathrm{CdSe}$ QDs as a $\mathrm{S}^{-}-\mathrm{COO}^{-}$bidentate ligand. 204 Density functional theory (DFT) calculations ${ }^{59}$ revealed that 205 the interaction patterns between Cys and $(\mathrm{CdSe})_{n}$ vary with 206 the cluster size and medium. Pattern $\mathrm{S} \rightarrow \mathrm{Cd} \leftarrow \mathrm{N}$ is preferred 207 in the gas phase, toluene, and alkaline solution, while pattern $\mathrm{O} 208$ $\rightarrow \mathrm{Cd}$ and $\mathrm{H} \rightarrow$ Se is preferred in water. In another study, ${ }^{60} 209$ DFT calculations were performed on complexes of $(\mathrm{CdSe})_{13} 210$ 
a)

b) $\bigcirc$ High Cys conc. $\bigcirc$ Low Cys conc.
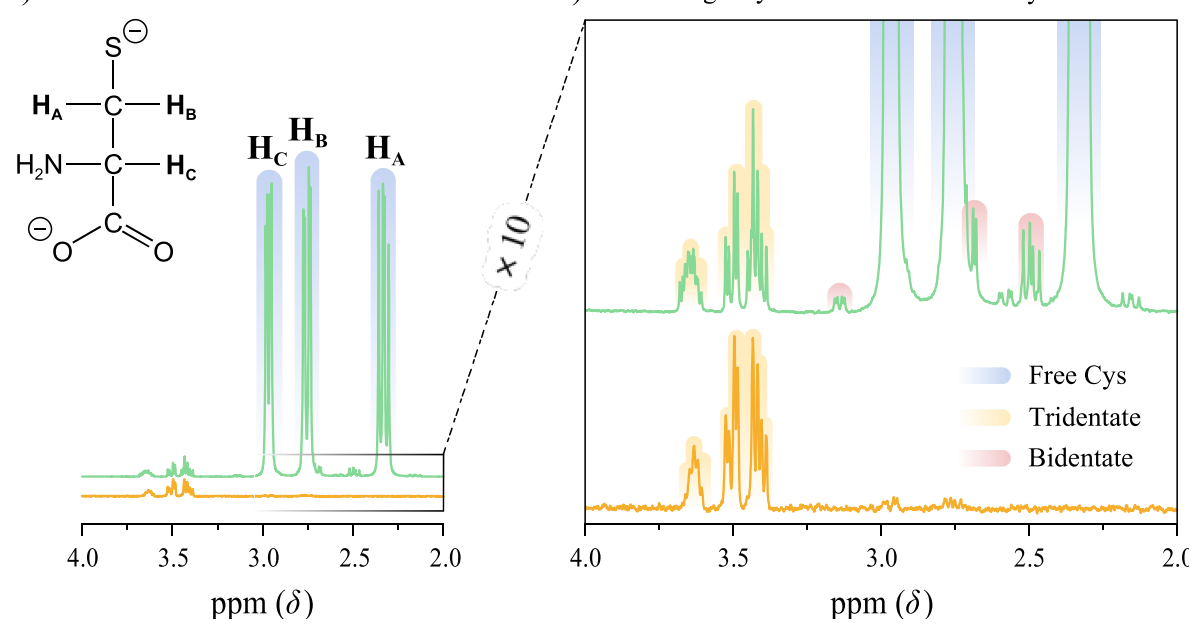

Figure 4. (a) ${ }^{1} \mathrm{H}$ NMR spectra of QDs in $\mathrm{H}_{2} \mathrm{O}$ with high and low Cys concentration at $\mathrm{pH} 13$ for free Cys, and (b) scaled-up ${ }^{1} \mathrm{H}$ NMR spectra of QDs with high and low Cys concentrations, including peaks of bound Cys.

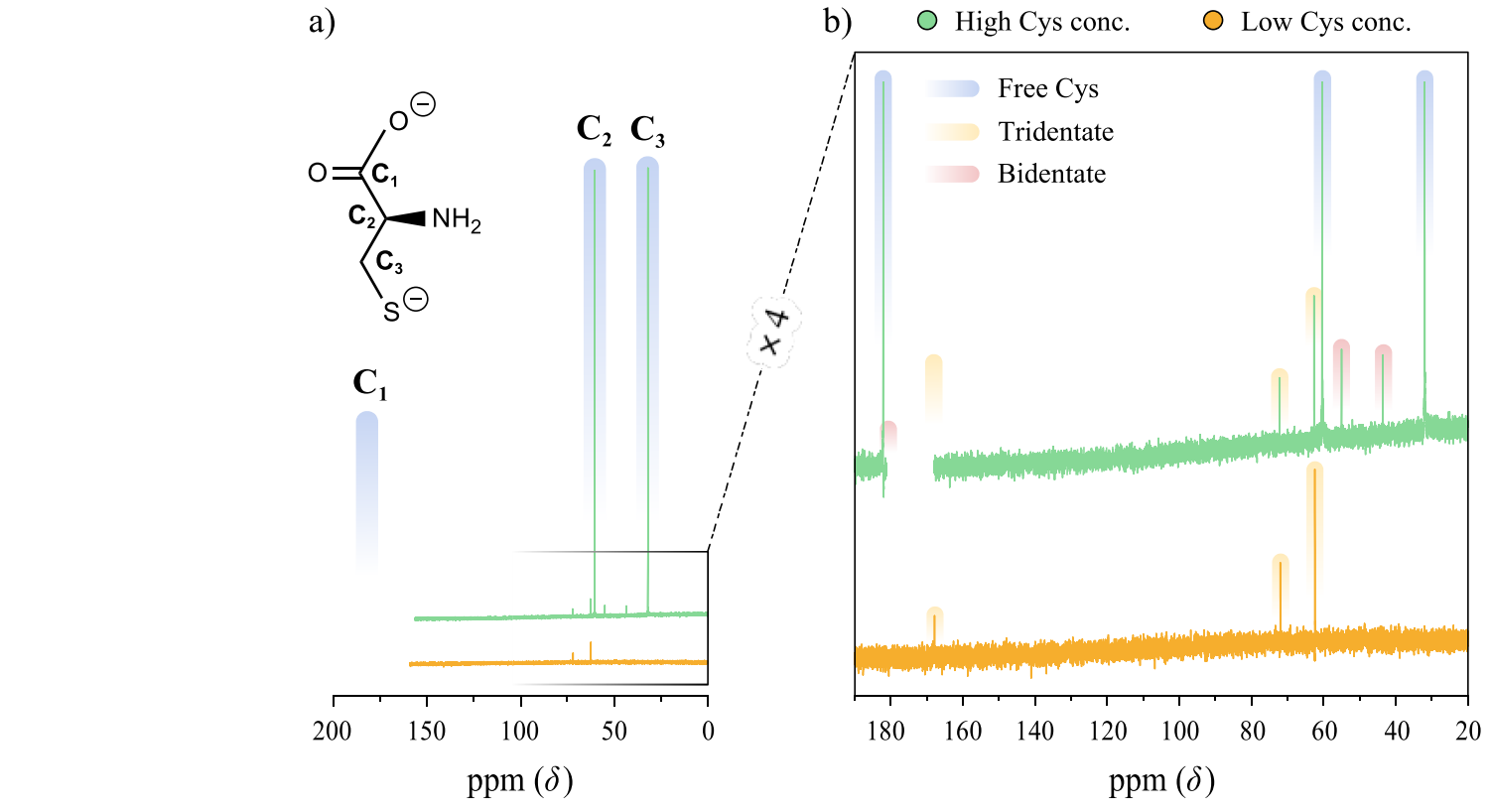

b) $\bigcirc$ High Cys conc. $\bigcirc$ Low Cys conc.

Figure 5. (a) ${ }^{13} \mathrm{C}$ NMR spectra of QDs aqueous solutions with high and low Cys concentration at $\mathrm{pH} 13$ for free $\mathrm{Cys}$, and (b) scaled-up ${ }^{13} \mathrm{C}$ NMR spectra of QDs with high and low Cys concentrations, including peaks of bound cysteine.

211 nanoclusters and Cys molecules attached as tridentate ligands 212 to the distorted surface of the nanoclusters. Hence, it is known 213 that binding modes of Cys are dependent on many factors such 214 as medium, $\mathrm{pH}$, cluster size, etc.

215 Previous studies on aqueous metal complexes have 216 demonstrated that the Cys binding mode is also dependent 217 on the ratio between the Cys and metal ions. For example, Cys 218 can be coordinated to $\mathrm{Pb}^{2+}$ ions in a tridentate fashion in 1:1 219 complexes, while in 2:1 complexes, it binds in a bidentate 220 fashion. $^{61}$ Prompted by these results, we suggested that the 221 concentration of Cys might influence its binding mode, and, 222 consequently, the $G$-factor intensity of Cys-stabilized QDs.

223 Investigation of Cys Binding Modes by NMR Spec224 troscopy. To investigate how the Cys concentration 225 influences its binding mode on the $\mathrm{QD}$ surface, ${ }^{1} \mathrm{H}$ and ${ }^{13} \mathrm{C}$ 226 NMR spectroscopy analyses of two different Cys QD solutions 227 were performed: (1) a solution with a "low" Cys concentration 228 (ca. $0.2 \mathrm{mg} / \mathrm{mL}$ ), at which G-factor increased, and (2) a solution with a "high" Cys concentration (ca. $20 \mathrm{mg} / \mathrm{mL}$ ), 229 corresponding to the region where the $G$-factor is minimum. 230 Cys-QD solutions for NMR analysis were prepared and 231 measured under argon atmosphere to avoid Cys oxidation. 232

${ }^{7} \mathrm{H}$ NMR Spectroscopy. The ${ }^{1} \mathrm{H}$ NMR spectrum of free Cys 233 at $\mathrm{pH} 13$ is different from one at neutral $\mathrm{pH} .{ }^{61}$ It has the form 234 of three double doublets (Figure 4a), corresponding to a $\mathrm{H}_{235} \mathrm{f4}$ atom bound to a $\mathrm{C}_{2}$ atom with a chemical shift of $2.97 \mathrm{ppm} 236$ $\left({ }^{1} \mathrm{H}_{\mathrm{C}}\right)$, and two $\mathrm{H}$ atoms bound to $\mathrm{C}_{3}$, which are chemically 237 inequivalent, with 2.33 and 2.75 ppm chemical shifts $\left({ }^{1} \mathrm{H}_{\mathrm{A}}\right.$ and 238 ${ }^{1} \mathrm{H}_{\mathrm{B}}$, respectively). The carboxylic and thiol groups are ${ }_{239}$ deprotonated at $\mathrm{pH} 13\left(\mathrm{p} K_{\mathrm{a}}=1.71\right.$ for $-\mathrm{COOH}$ and $\mathrm{p} K_{\mathrm{a}}=240$ 8.27 for $-\mathrm{SH}),{ }^{62}$ and accordingly, the ${ }^{1} \mathrm{H}$ NMR spectra did not 241 display any signals arising from protons on these groups. We 242 note that the ${ }^{1} \mathrm{H}$ NMR peak of the amino group is not usually 243 observed in aqueous solutions. $^{63,64}$ 244 


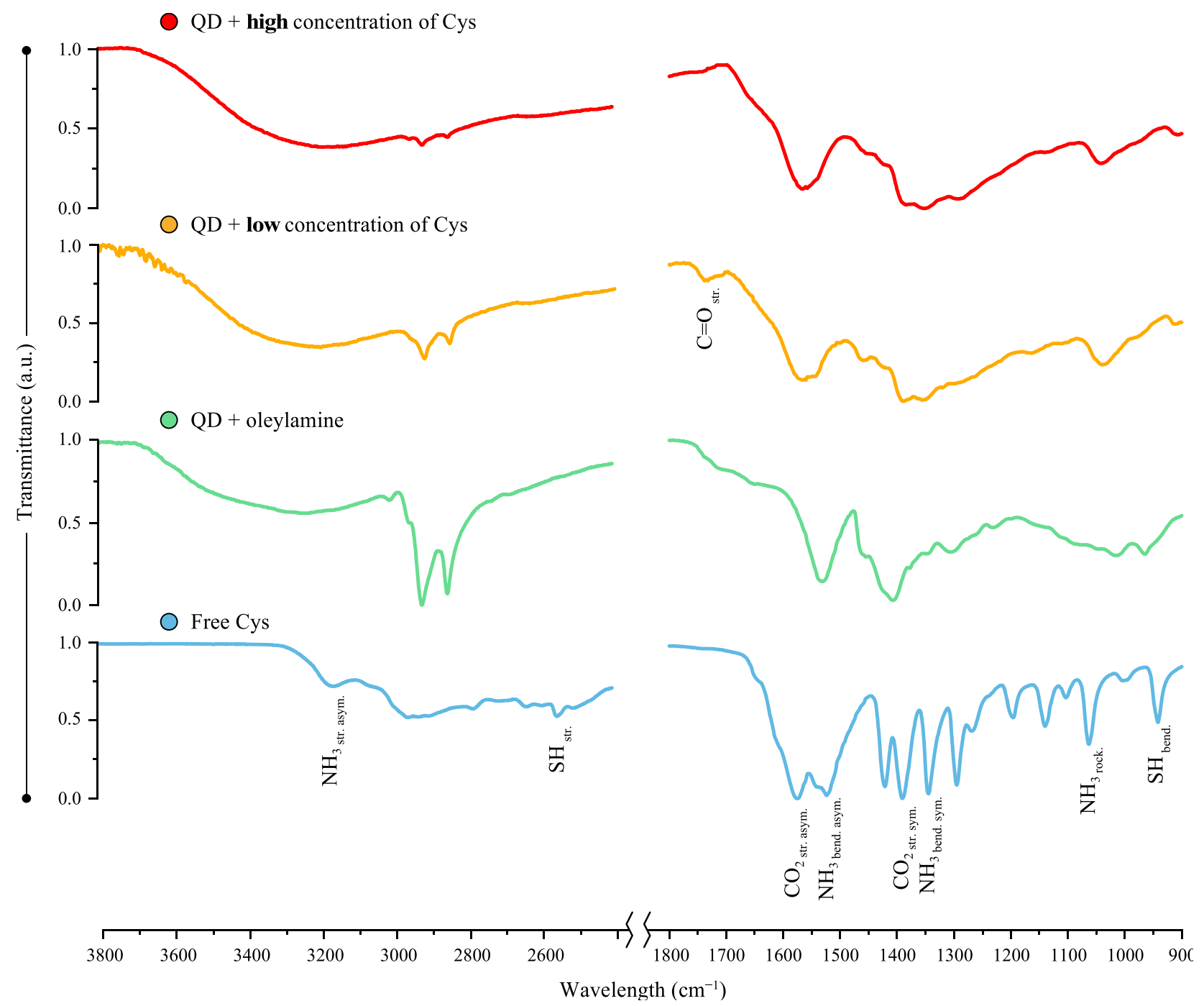

Figure 6. FTIR spectra of QDs precipitated from aqueous solutions with a high and low Cys concentration, QDs with organic ligandsmostly oleylamine and free cysteine. Full FTIR spectra are presented in Figure S4 in the Supporting Information.

245 At a low Cys concentration, almost all ligands are bound to 246 the QD surface, and only one set of peaks corresponding to the 247 tridentate ${ }^{61}$ binding mode of the molecules is observed in the ${ }_{248}{ }^{1} \mathrm{H}$ NMR spectrum. This can be seen in Figure $4 \mathrm{~b}$ (orange 249 line), where peaks were shifted downfield, compared to free 250 Cys $\left({ }^{1} \mathrm{H}_{\mathrm{A}},{ }^{1} \mathrm{H}_{\mathrm{B}}\right.$, and ${ }^{1} \mathrm{H}_{\mathrm{C}}$ shifted from 2.33, 2.75, and $2.97 \mathrm{ppm}$ 251 to $3.42,3.50$ and $3.63 \mathrm{ppm}$, respectively). ${ }^{61}$ At a high Cys 252 concentration, three sets of ${ }^{1} \mathrm{H}$ NMR peaks were observed 253 (Figure 4b, green line): (i) the first intense peak set 254 corresponds to the free Cys; (ii) the second one presents 255 the same peaks associated with the tridentate mode registered 256 in the spectrum at low Cys concentration; and (iii) the third 257 one corresponds to the bidentate form. In the case of the 258 bidentate mode, the ${ }^{1} \mathrm{H}_{\mathrm{B}}$ peak is shifted downfield, with respect 259 to the free Cys form, which corresponds to the $\mathrm{S}^{-}$binding to $260 \mathrm{Cd}^{2+}$, while the ${ }^{1} \mathrm{H}_{\mathrm{C}}$ peak did not shift very much, compared to 261 the tridentate form, indicating that the $\mathrm{COO}^{-}$is most likely 262 free.

${ }^{263}{ }^{13} \mathrm{C} N M R$ Spectroscopy. ${ }^{13} \mathrm{C}$ NMR results were consistent 264 with the ${ }^{1} \mathrm{H}$ NMR data. Each Cys molecule has three carbon 265 atoms, with the following ${ }^{13} \mathrm{C}$ NMR peaks: ${ }^{13} \mathrm{C}_{1}$, representing 266 the $\mathrm{C}$ atom of the carboxylic group; ${ }^{13} \mathrm{C}_{2}$ and ${ }^{13} \mathrm{C}_{3}$, 267 representing the $\mathrm{C}$ atoms bound to $\mathrm{NH}_{2}$ and $\mathrm{S}^{-}$groups with the peak positions at 179,59 , and $29 \mathrm{ppm}$, respectively (Figure $268 \mathrm{fs}$ $5 \mathrm{a}$ ). At low concentrations (Figure 5b, orange lines), only one $269 \mathrm{fs}$ set of Cys peaks (different from that of the free molecule) can 270 be observed, indicating that almost all cysteine molecules are 271 bound to the QD surface. Besides, all three signals present a 272 significant shift in position (from $179 \mathrm{ppm}$ to $168 \mathrm{ppm}$ for the 273 carboxylic ${ }^{13} \mathrm{C}_{1}$, ${ }^{65}$ from $59 \mathrm{ppm}$ to $72 \mathrm{ppm}$ and from $29 \mathrm{ppm}$ to 274 $62 \mathrm{ppm}$ for the aminated ${ }^{13} \mathrm{C}_{2}$ and the thiolated ${ }^{13} \mathrm{C}_{3}, 275$ respectively), ${ }^{66}$ suggesting that, at low concentrations, Cys is 276 coordinated in a tridentate mode.

At the higher Cys concentration (Figure 5b, green lines), the 278 ${ }^{13} \mathrm{C}$ NMR spectrum presents three sets of peaks: (i) the first 279 one, with three intense signals at the same positions as free 280 Cys; (ii) the second one, with three weak peaks corresponding 281 to the low concentration ones; and (iii) the third one, where 282 ${ }^{13} \mathrm{C}_{1}$ remains almost in the same position, but ${ }^{13} \mathrm{C}_{2}$ and ${ }^{13} \mathrm{C}_{3}{ }_{283}$ are shifted from $59 \mathrm{ppm}$ to $55 \mathrm{ppm}$ and from $29 \mathrm{ppm}$ to 44284 ppm, respectively. Similar shifts have been reported for a 285 $\mathrm{NH}_{2}-\mathrm{SH}$ bidentate mode; ${ }^{67}$ therefore, we attribute these 286 signals to Cys molecules that are bound to the QD surface in a 287 $\mathrm{S}^{-}-\mathrm{NH}_{2}$ bidentate configuration. ${ }^{67} 288$

Investigation of Cys Binding Modes by FTIR Spec- 289 troscopy. To determine the binding mode of the amino 290 
291 group, which was not obvious from NMR analysis, we have 292 also performed FTIR analysis of our QD samples. To prepare 293 the samples for our FTIR studies, Cys-stabilized QDs were 294 precipitated from aqueous solutions with high and low Cys 295 concentrations, using acetone, and subsequently dried. It is 296 important to note that samples did not contain water, which 297 has a very broad and intense peak in the $3000-3750 \mathrm{~cm}^{-1}$ 298 region that overlaps with the asymmetrical stretching vibration 299 peaks of the amino group. Cys-QDs spectra are provided in 300 Figure 6 for comparison with QDs functionalized with initial 301 organic ligands (mostly oleylamine) and with pure Cys.

302 The $\mathrm{S}^{-}$group of free Cys has two peaks at 2550 and 942 $303 \mathrm{~cm}^{-1}$, corresponding to its stretching and bending modes, 304 respectively. Both peaks are absent in the spectra of Cys-QDs. 305 The $\mathrm{NH}_{2}$ group of free Cys has a peak associated with 306 asymmetrical stretching vibrations at $3165 \mathrm{~cm}^{-1}$, which is 307 significantly broadened when $\mathrm{NH}_{2}$ is bound to the QD surface. 308 This broadening can be observed in the spectra of QDs with 309 oleylamine and QDs with Cys, both at high and low 310 concentrations, confirming that the amino group is coordi311 nated with the QD surface. Pure Cys has a zwitterionic form 312 with a deprotonated carboxylic group $\left(\mathrm{COO}^{-}\right)$and a 313 protonated amino group $\left(\mathrm{NH}_{3}{ }^{+}\right)$. The free negatively charged 314 carboxylic group has asymmetric and symmetric stretching 315 vibration peaks at 1575 and $1391 \mathrm{~cm}^{-1}$, respectively. The 316 appearance of a carbonyl stretching mode peak at $1735 \mathrm{~cm}^{-1}$ 317 on the FTIR spectra of QDs with a low Cys concentration 318 indicates the binding of the $\mathrm{COO}^{-}$group. QDs with a high 319 amount of Cys ligands display a smaller peak at this area, 320 indicating that part of the $\mathrm{COO}^{-}$groups are free. This is in full 321 agreement with the NMR data.

322 Effect of Cys Binding Mode on the Ligand Config323 uration on the QD Surface. The Cys molecule has three 324 staggered rotamers as the result of rotation along the $\mathrm{C}_{2}-\mathrm{C}_{3}$ 325 axis (a), namely, a trans-rotamer, where the carboxyl and thiol 326 groups are in trans position, and two gauche-rotamers, where 327 these groups are separated by a torsion angle of $60^{\circ}$ (see 328 Figure $7 \mathrm{a})$.

329 Previous reports of NMR studies of $\mathrm{Pb}^{\mathrm{II}}$-based Cys 330 complexes have shown that, under alkaline conditions, when $331 \mathrm{COOH}$ and $\mathrm{SH}$ groups of free $\mathrm{Cys}$ are deprotonated and

a)

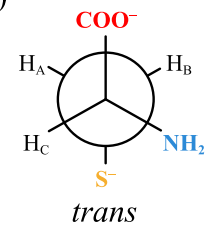

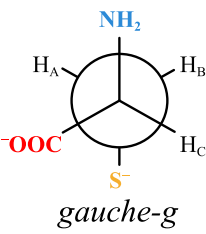

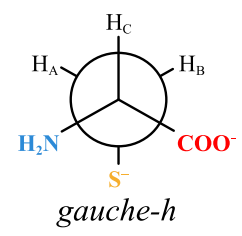

b)

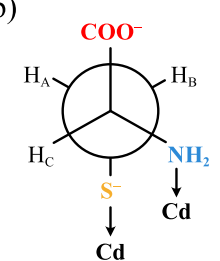

$\mathrm{S}^{-}-\mathrm{NH}_{2}$ bidentate

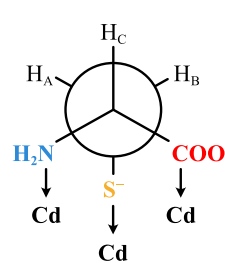

Tridentate
Figure 7. (a) Newman projections of the three rotamers of the Cys molecule. (b) Newman projections of $\mathrm{S}^{-}-\mathrm{NH}_{2}$ bidentate (left) and tridentate (right) coordination modes of Cys on the QD surface. negatively charged, the trans rotamer is prevalent. ${ }^{61}$ Therein, it 332 was also demonstrated that if the Cys ligand is bound to $\mathrm{Pb} 333$ metal ion through all three functional groups, NMR spectra 334 mostly correspond to the gauche- $h$ rotamer, i.e., all three 335 groups are located at one side of the molecule, which facilitates 336 tridentate Cys binding. When Cys is coordinated via a $\mathrm{S}^{-}-337$ $\mathrm{NH}_{2}$ bidentate mode, both conformations are present in 338 solution, but the trans rotamer is prevalent. However, in our 339 system, when Cys is bound to the QD surface, the trans 340 rotamer can be even more preferable, because of steric 341 constraints.

Other previous studies have shown that one type of 343 enantiomer of a chiral molecule can bind to the nanoparticle 344 surface in different ways. ${ }^{28,55}$ For example, acetyl-L-Cys can 345 bind to $\mathrm{HgS}$ via the $\mathrm{SH}$ and $\mathrm{COOH}$ groups or $\mathrm{SH}$ and $\mathrm{Ac} 346$ groups, depending on the synthesis conditions. ${ }^{55}$ Sometimes, 347 these bound molecules exhibit almost mirror-image config- 348 urations on the QD surface, which gives rise to inverted CD 349 spectra. These results were further confirmed by theoretical 350 calculations. $^{28}$

In our study, Cys can bind to the QD surface in a tridentate 352 and $\mathrm{S}^{-}-\mathrm{NH}_{2}$ bidentate fashion, as was shown above, based on 353 FTIR and NMR data, and Cys molecules in these two 354 coordination modes can adopt different rotamers: the gauche- $h 355$ rotamer for the tridentate binding mode, and, prevalently, the 356 trans rotamer for the bidentate coordination (see Figure $7 \mathrm{~b}$ ). 357 These two rotamer forms bound to the QD can be considered 358 as almost diastereomers of each other, with respect to the 359 relative position of the $\mathrm{S}^{-}$and the $\mathrm{NH}_{2}$ groups. Hence, we 360 suggest that, because of these diastereomeric conformations, 361 Cys bound in bidentate and tridentate modes can be the origin 362 of the opposite CD signals. Therefore, at high concentrations, 363 when the amount of Cys molecules bound in a bidentate mode 364 increases, the $\mathrm{CD}$ signal produced by the tridentate ligands at 365 low concentrations is partially compensated, resulting in an 366 overall decrease of the CD signal.

Computational Studies of Cys Binding Modes. Based 368 on the experimental observations presented above, a likely 369 explanation for the change in the coordination of the adsorbed 370 Cys ligands upon increase of Cys concentration is the 371 emergence of ligand-ligand interactions. To confirm this, we 372 conducted a thorough investigation by means of periodic DFT 373 calculations (see the Materials and Methods section for 374 details) in order to elucidate the binding modes of Cys 375 molecules on the QD surface at different concentrations. 376 Following an exhaustive iterative coverage analysis, we selected 377 five theoretical models featuring 1-5 Cys molecules adsorbed 378 on a $p(3 \times 3)$ supercell of a $\mathrm{CdS}(0001)$ surface- 379 corresponding to the structure of the exposed QD shell- 380 with the objective of reproducing low, intermediate, and high 381 coverage limits, respectively. For each surface coverage, we 382 then considered all possible combinations of binding modes, 383 rendering a total of 67 different structures. The results 384 obtained for the most energetically favorable adsorptions are 385 summarized in Figure 8, which also displays the binding modes $386 \mathrm{f} 8$ adopted by each of the ligands per unit cell.

387

The analysis of the most stable configurations of the low- 388 coverage model (Figure 8a) revealed that Cys ligands are 389 predominantly bound to the QD surface in a tridentate 390 conformation with the $\mathrm{S}^{-}, \mathrm{COO}^{-}$, and $\mathrm{NH}_{2}$ groups 391 coordinated to surface $\mathrm{Cd}^{2+}$ ions, in agreement with experi- 392 ments. As can be seen in Figure 8b, the addition of a second 393 ligand did not affect the Cys binding mode in the lowest 394 
a)

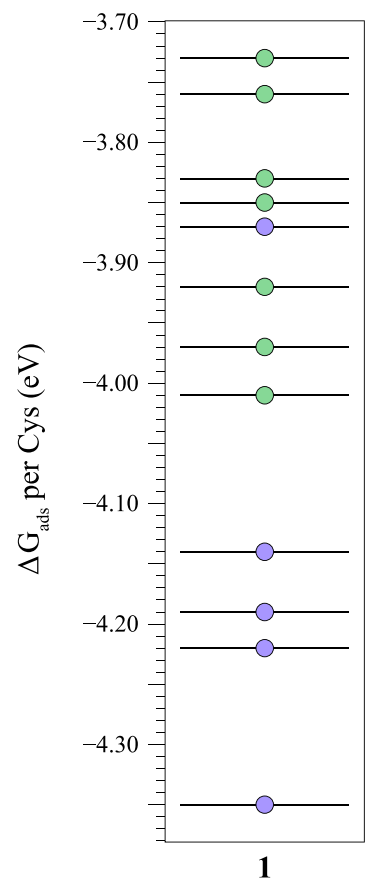

b)

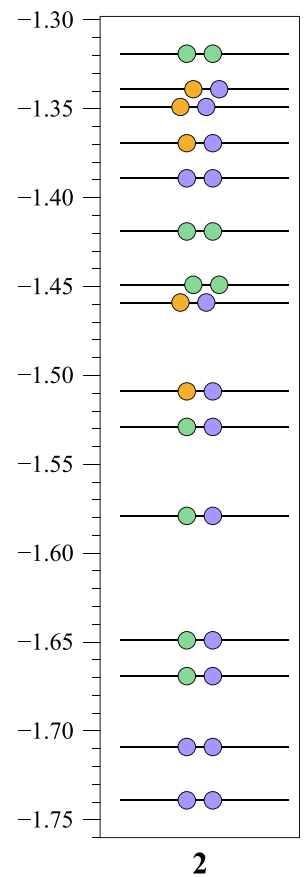

- Monodentate c)

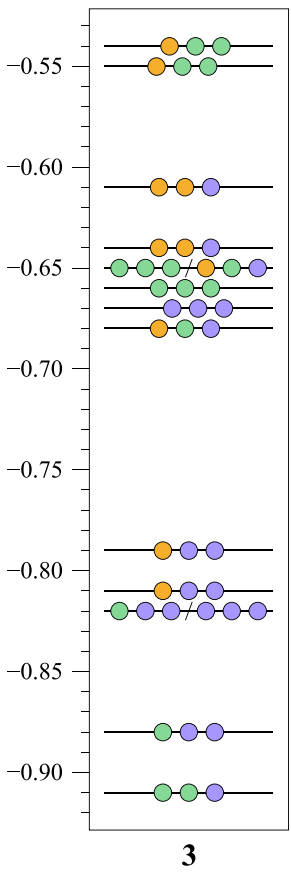

Number of adsorbed Cys

- Bidentate d)

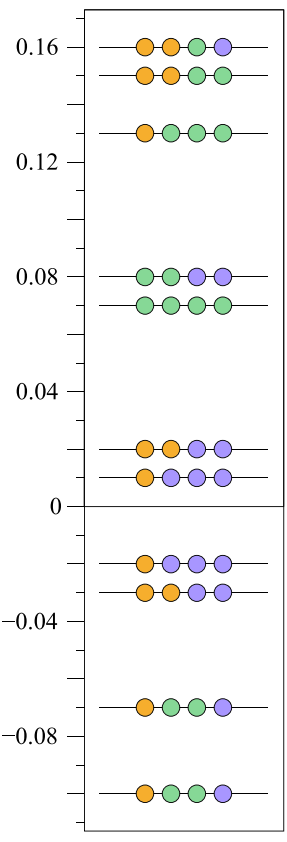

4 e)

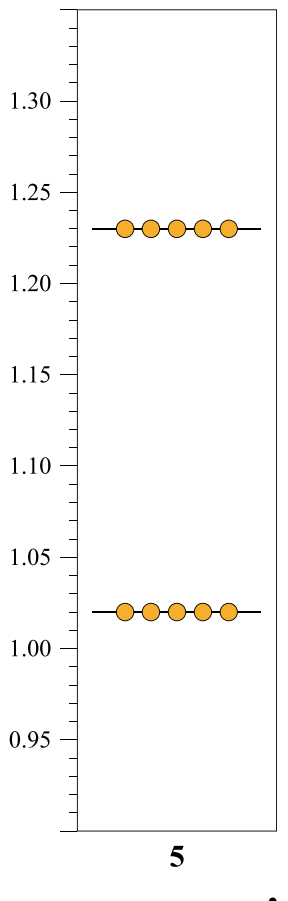

- Tridentate

Figure 8. Calculated adsorption Gibbs energies, $\Delta G_{a d s}$, for the (a) low, (b, c) intermediate, and (d, e) high coverage models with $1-5$ Cys molecules per unit cell. Each line represents a different configuration of the ligands within a given coverage. Monodentate, bidentate, and tridentate Cys binding modes are represented as orange, green, and purple circles, respectively. Gray areas indicate endergonic configurations; therefore, these are predicted to be unlikely. We note that, in panel (c), there are two sets of configurations that possess the same $\Delta G_{\text {ads }}$ value, up to the second decimal digit; these are represented on the same line and separated by a slash.

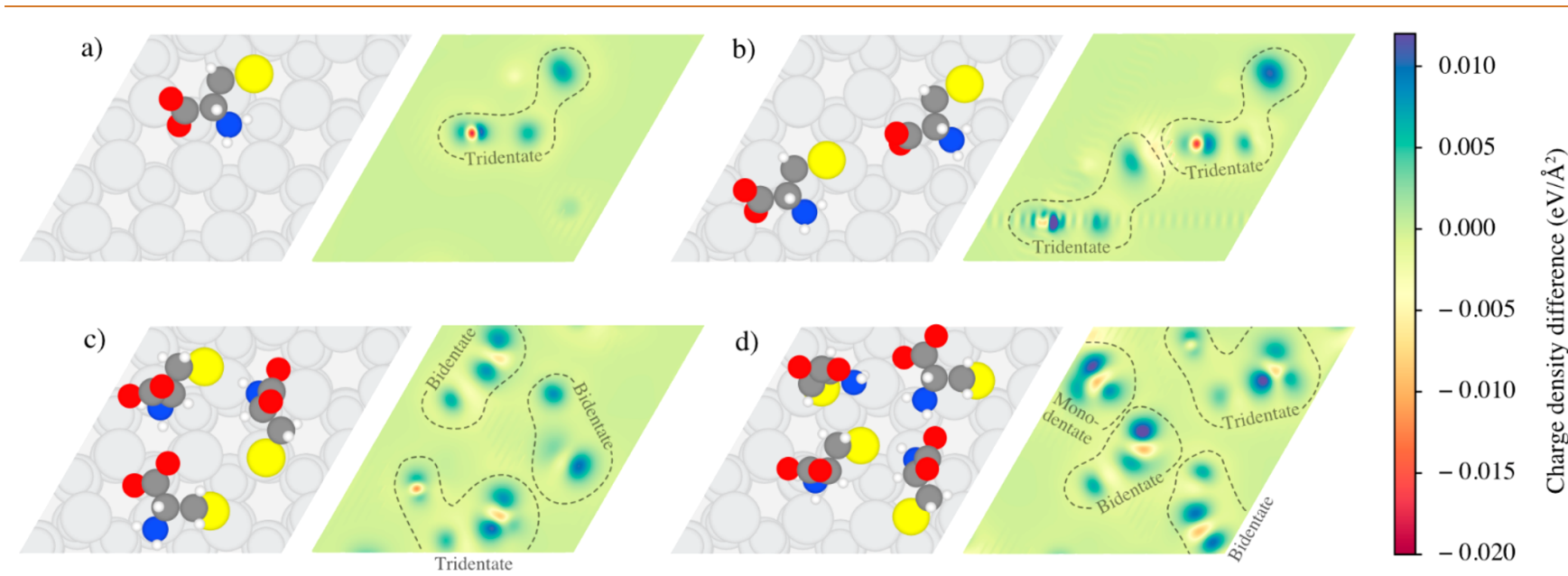

Figure 9. Optimized structures of the most stable configurations with a predicted exergonic adsorption for the (a) low, (b, c) intermediate, and (d) high coverage models featuring 1-4 Cys, alongside a 2D plot of the charge density difference across the interaction plane. [Atom color code: C (gray), H (white), $\mathrm{N}$ (blue), $\mathrm{O}$ (red), and S (yellow).]

395 energy configuration. However, DFT calculations predict the 396 emergence of a relatively stable configuration (only $70 \mathrm{meV}$ 397 higher in energy, with respect to the lowest energy 398 configuration) in which one of the Cys ligands becomes 399 bidentate through the $\mathrm{S}^{-}$and $\mathrm{NH}_{2}$ groups, while the other one 400 remained coordinated in a tridentate mode. This original 401 configuration points toward a decrease in the prevalence of the 402 tridentate mode at higher Cys coverages, which, again, is 403 consistent with experimental observations. This was indeed 404 observed upon the addition of the third and fourth Cys ligands in the intermediate and high coverage models depicted in 405 Figures $8 \mathrm{c}$ and $8 \mathrm{~d}$. In both cases, the bidentate conformation 406 was found to predominate in the most stable configurations, 407 where even a monodentate Cys was predicted to coexist on the 408 surface covered with four ligands. An inclusion of a fifth Cys 409 molecule was revealed to be energetically unfeasible based on 410 the endergonic $\Delta G_{\text {ads }}$ values obtained for the models depicted 411 in Figure 8e. The differential adsorption of the Cys in each 412 coverage model is depicted in Figure S5 in the Supporting 413 Information. 
a)

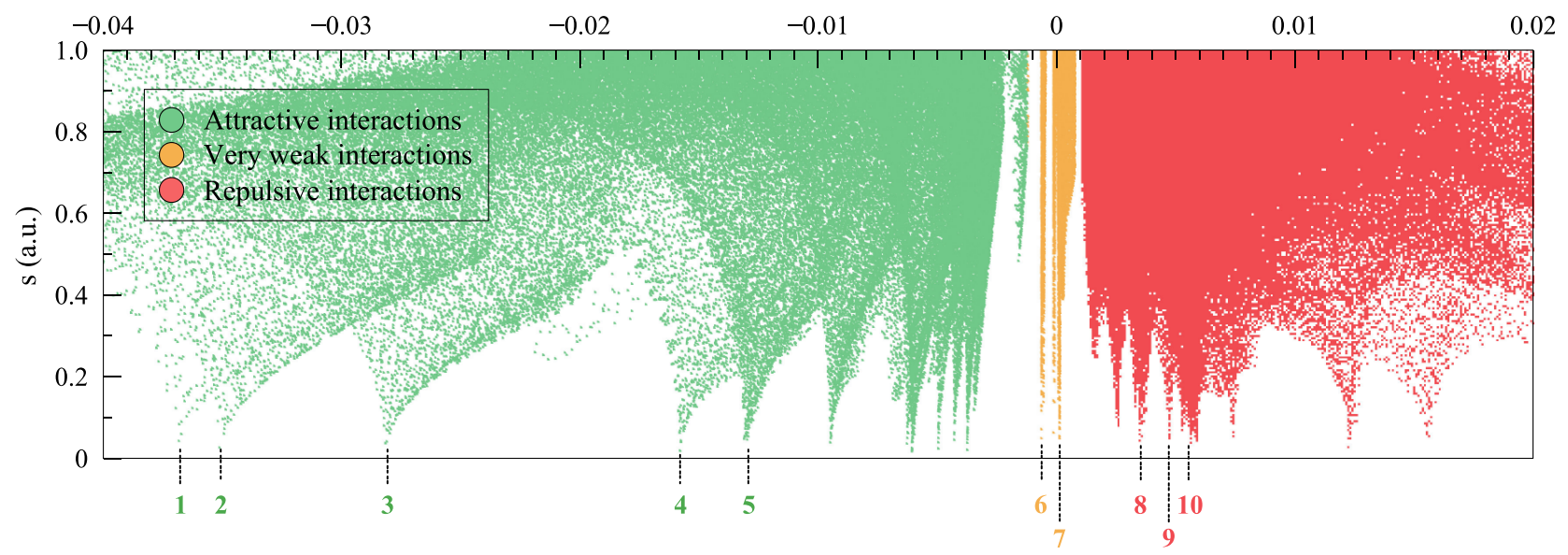

b)

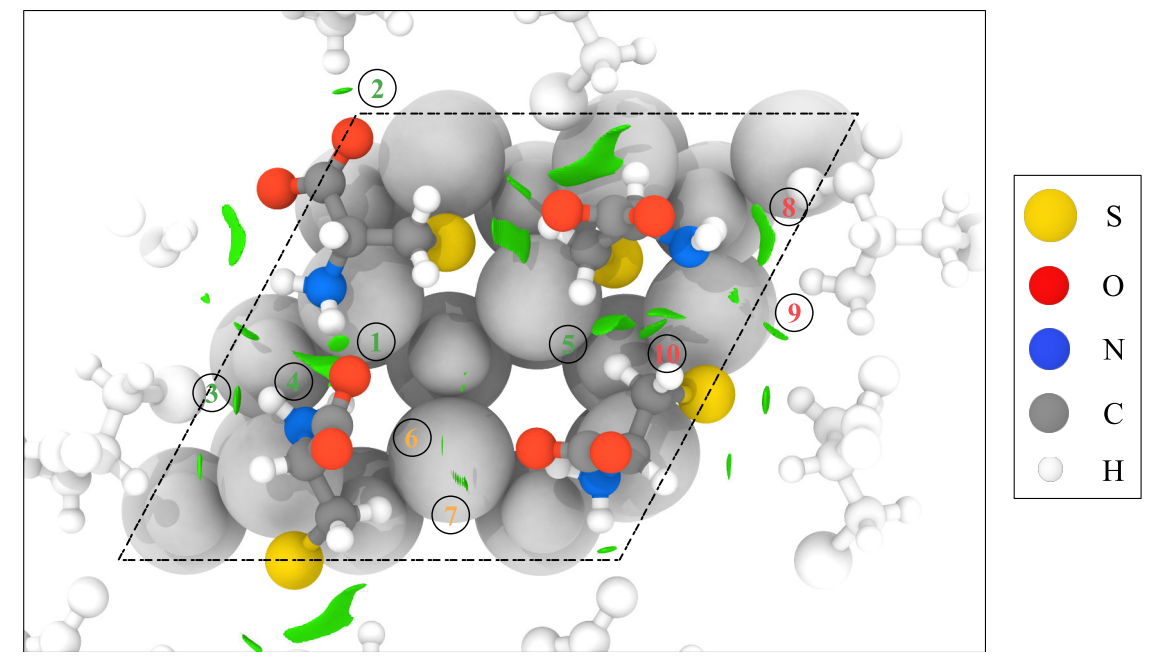

Figure 10. (a) Plot of $s$, as a function of $\operatorname{sign}\left(\lambda_{2}\right) \rho$. (b) Representation of the intermolecular noncovalent interactions inside a unit cell, displayed as a green isosurface with an isovalue of $0.45 \mathrm{e}^{-} /(\text {a.u. })^{3}$. The 10 most relevant interactions are labeled in both the reduced density gradient plot and the representation of the noncovalent interactions. Atoms of Cys ligands from neighboring unit cells are colored white, while $\mathrm{Cd}$ and $\mathrm{S}$ atoms from the QD surface are colored light gray for clarity.

415 The lowest energy structures from the coverages including 416 1-4 Cys ligands are represented in Figure 9, alongside two417 dimensional (2D) plots of the charge density differences 418 between the ligands and the QD surface. In these plots, 419 warmer (orange and red) and colder (turquoise and blue) 420 colors indicate a decrease and increase, respectively, in the 421 charge density difference across the interaction plane. Hence, 422 functional groups that lay on higher or lower charge-density 423 difference regions are predicted to interact with the QD 424 surface. This allows one to confirm the binding mode of each 425 adsorbed Cys molecule, based on the number of functional 426 groups laying on these regions.

427 To understand the change in the Cys binding mode 428 observed from tridentate to bidentate and monodentate at 429 high ligand concentrations, we subsequently analyzed the 430 intermolecular noncovalent interactions (NCIs) on QD 431 surfaces terminated with 2-5 Cys molecules (see the Materials 432 and Methods section for details) - the low coverage model 433 with 1 Cys per unit cell was not considered, since ligands in 434 periodic images are too far apart to interact noncovalently. 435 Importantly, the methodology employed herein allows for the 436 semiquantitative analysis of these interactions by identifying 437 the regions in which the atomic clouds of the Cys molecules overlap. In short, since the electron density in these regions is 438 maximum, the reduced density gradient approaches zero. 439 Hence, by plotting the reduced density gradient $(s)$ as a 440 function of the density multiplied by the sign of the second 441 eigenvalue of the Hessian matrix $\operatorname{sign}\left(\lambda_{2}\right) \rho$, which effectively 442 distinguishes if the interaction is attractive [negative] or 443 repulsive [positive]), a series of peaks are obtained, which can 444 be attributed to each NCI and its attractive or repulsive 445 behavior, as described elsewhere. ${ }^{68,69}$ Attractive interactions 446 include hydrogen bonding, dipole-dipole, and London 447 dispersion interactions, while very weak interactions corre- 448 spond to long-range van der Waals interactions; repulsive 449 interactions mainly encompass steric effects.

The NCI analysis of the high coverage model with 4 Cys per 451 unit cell is displayed in Figure 10. According to this $452 \mathrm{f} 10$ representation, the three strongest attractive interactions 453 correspond to the hydrogen bonding between the $\mathrm{NH}_{2} 454$ group and $\mathrm{COO}^{-}$or $\mathrm{S}^{-}$. Interestingly, these attractive 455 interactions offset the steric clashes between Cys molecules, 456 making this configuration stable with a $\Delta G_{\text {ads }}$ per Cys of ca. 457 $-0.10 \mathrm{eV}$ (Figure $8 \mathrm{~d}$ ). However, the ability of Cys molecules 458 to create such strong attractive interactions is hindered upon 459 the addition of a fifth Cys (high coverage, Figure 8e), which is 460 
461 derived in weaker attractive interactions and stronger steric 462 clashes leading to the unfavorable adsorption of further Cys 463 ligands. The NCI plots for both low and high coverage models 464 are presented in Figure S6 in the Supporting Information.

465 Overall, DFT calculations clearly show that, at low 466 concentrations, Cys molecules are predominantly adsorbed 467 on the $\mathrm{Cd}^{2+}$ ions of the QD surface in a tridentate mode via the $468 \mathrm{~S}^{-}, \mathrm{NH}_{2}$, and $\mathrm{COO}^{-}$groups. However, this trend in 469 coordination is altered as the Cys concentration increases. 470 Particularly, the high coverage models predict the prevalence 471 of bidentate Cys ligands bound via the $\mathrm{S}^{-}$and $\mathrm{NH}_{2}$ groups, 472 with the possibility of ligands present in a monodentate 473 coordination via the $\mathrm{S}^{-}$moiety. These results are in good 474 agreement with the experimental data observed in the NMR 475 and FTIR spectra at low and high Cys concentrations, showing 476 the same trend in the coordination change with concentration.

\section{CONCLUSIONS}

478 Thus, in this work the influence of chiral Cys ligand 479 concentration on the chiroptical response intensity of $\mathrm{CdSe} /$ $480 \mathrm{CdS}$ QDs has been investigated. Experiments demonstrated 481 that QD CD signal intensity increases with Cys concentration 482 at the beginning, then reaches a maximum and decreases at 483 high Cys concentrations. We found that the intensity of CD 484 signal showed a 10 -fold increase at an optimal Cys 485 concentration of $0.26 \mathrm{mg} / \mathrm{mL}$. NMR and FTIR analyses 486 demonstrated that Cys molecules adopt different binding 487 configurations on the $\mathrm{QD}$ surface at different ligand 488 concentrations. Particularly, at high concentrations, Cys 489 molecules are most likely bound to the QD surface via the $490 \mathrm{~S}^{-}$and $\mathrm{NH}_{2}$ groups, whereas at low concentrations, they are 491 coordinated via all three functional groups, i.e., $\mathrm{S}^{-}, \mathrm{NH}_{2}$ and $492 \mathrm{COO}^{-}$. Our results also suggest that tridentate and bidentate 493 configurations of bound Cys are almost diasterioisomeric 494 configurations, which gives rise to opposite CD signals. At high 495 Cys concentrations, however, at which large amounts of Cys 496 are bound in a bidentate mode, the CD signal can decrease as a 497 result of superposition of those opposite CD signals. These 498 results were fully supported by our DFT calculations, which 499 indicate a clear change in the coordination mode of Cys 500 molecules as the ligand concentration increases. Furthermore, 501 simulations indicated that variations in the binding modes are 502 caused by noncovalent interactions between the ligands. 503 Overall, this combined experimental and theoretical work 504 demonstrated that chiroptical properties of QDs are strongly 505 dependent on the concentration and binding modes of chiral 506 ligands, which is very important for the understanding of 507 chiroptical phenomena at the nanoscale and the future design 508 of advanced optically active nanomaterials. Since chirality plays 509 a key role in chemical and biological systems, the results 510 described herein are of considerable interest, from both a 511 fundamental and practical point of view, and may usefully 512 contribute to the development of potential applications of 513 optically active nanocrystals, including optical chiral sensing, 514 detection of various enantiomeric species, enantiospecific 515 separation, asymmetric catalysis, and biological imaging.

\section{MATERIALS AND METHODS}

517 Chemicals. All chemical reagents were of analytical grade and used 518 as purchased without further purification. L-Cys and D-Cys, HPLC 519 acetone, HPLC hexane, HPLC toluene, oleic acid (90\%), 1520 octadecene (ODE, 90\%), selenium (99.99\%), sulfur (99.998\%), 521 oleylamine (98\%), trioctylphosphine (TOP, 97\%), and trioctylphos- phine oxide (TOPO, 99\%) were purchased from Sigma-Aldrich. 522 Cadmium oxide (99.995\%) were purchased from Alfa Aesar. 523 Hydrochloric acid, methanol, chloroform, and potassium hydroxide 524 were purchased from Sigma-Aldrich and used for phase-transfer 525 procedure. Toluene and distilled water (Millipore) were used as 526 solvents.

Synthesis of CdSe QDs. CdSe QDs were synthesized following 528 the protocol described in a previous work. ${ }^{33}$ A $0.2 \mathrm{M}$ Cd-oleate stock 529 solution (in ODE) was prepared by adding $0.257 \mathrm{~g}$ of $\mathrm{CdO}$ to $2 \mathrm{~mL} 530$ of oleic acid in $8 \mathrm{~mL}$ of ODE, degassing it under reduced pressure, 531 and then heating to $300{ }^{\circ} \mathrm{C}$ under argon, followed by cooling to 30532 ${ }^{\circ} \mathrm{C}$. A $1.5 \mathrm{M}$ Se-TOP solution was prepared by dissolving $0.3553 \mathrm{~g}$ of 533 $\mathrm{Se}$ in $3 \mathrm{~mL}$ of TOP, using sonication under argon. Next, CdSe core 534 nanocrystals were prepared by mixing $4.5 \mathrm{~g}$ of octadecylamine, $1.5 \mathrm{~g} 535$ of TOPO, $12 \mathrm{~g}$ of ODE, and $3 \mathrm{~mL}$ of Cd-oleate solution in a three- 536 neck round-bottom flask. This mixture was then degassed at $90{ }^{\circ} \mathrm{C}$ for 537 $30 \mathrm{~min}$, flashed with argon, and then heated to $290{ }^{\circ} \mathrm{C}$. Upon 538 reaching this temperature, the Se-TOP solution above was injected 539 and the reaction vessel was immediately removed from the heat. The 540 solution was allowed to cool to room temperature, followed by the 541 addition of $20 \mathrm{~mL}$ of acetone to the mixture to isolate CdSe QDs. $\quad 542$

Synthesis of CdSe/CdS QDs. This synthesis was also performed 543 as reported in a previous work. ${ }^{33}$ The volumes used were calculated 544 using the SILAR approach to control the precise thickness of CdS 545 deposited. Initially, a $0.1 \mathrm{M}$ cadmium stock solution was prepared by 546 adding $0.514 \mathrm{~g}(4 \mathrm{mmol})$ of $\mathrm{CdO}$ to $8 \mathrm{~mL}$ of oleic acid and $32 \mathrm{~mL}$ of 547 ODE, which was degassed, then heated to $300{ }^{\circ} \mathrm{C}$ under argon, and 548 finally cooled to $30^{\circ} \mathrm{C}$. A $0.1 \mathrm{M}$ sulfur stock solution was prepared by 549 dissolving $0.128 \mathrm{~g}(4 \mathrm{mmol})$ of $\mathrm{S}$ in $40 \mathrm{~mL}$ of $\mathrm{ODE}$ at $180{ }^{\circ} \mathrm{C}$ under 550 argon (should appear as a yellow solution, changing from a very light 551 straw color at $120{ }^{\circ} \mathrm{C}$ ). Next, $33.2 \mathrm{~mL}$ of oleylamine, $66.4 \mathrm{~mL}$ of 552 ODE, and $2.24 \times 10^{-6} \mathrm{~mol}$ of QDs were added to a $250-\mathrm{mL}$ three- 553 neck round-bottom flask. This was heated to $50{ }^{\circ} \mathrm{C}$ and degassed for 554 $60 \mathrm{~min}$, followed by injection of $5.7 \mathrm{~mL}$ of Cd stock solution and, 555 finally, heating to $230{ }^{\circ} \mathrm{C}$. After $10 \mathrm{~min}$ at this temperature, $5.7 \mathrm{~mL}$ of 556 $\mathrm{S}$ solution was injected, followed by a $10 \mathrm{~min}$ wait. The reaction 557 mixture was then heated to $250{ }^{\circ} \mathrm{C}$ for $1 \mathrm{~h}$ to fully allow the reaction 558 to complete the growth of the first shell. At this time, $25 \mathrm{~mL}$ of the 559 reaction solution was removed and allowed to cool under argon, 560 producing the first sample. Subsequently, following the same 561 procedure, $6.2 \mathrm{~mL}$ of $0.1 \mathrm{M} \mathrm{Cd}$ and S stock solution was added to 562 grow the second shell, removing $25 \mathrm{~mL}$ of reaction solution again to 563 produce the second shell sample. This overall procedure was repeated 564 three times, injecting $6 \mathrm{~mL}$ for shell 3, $5.2 \mathrm{~mL}$ for shell 4, and $3.85 \mathrm{~mL} 565$ for shell 5 , following which the solution was allowed to cool to room 566 temperature, at which time $20 \mathrm{~mL}$ of acetone was added to precipitate 567 the samples using centrifugation. The precipitate was redispersed in a 568 minimum volume of hexane and then precipitated using acetone. This 569 procedure was repeated twice to produce a cleaned QD sample. $\quad 570$

Ligand Exchange of the CdSe/CdS Core/Shell Quantum 571 Dots with Chiral Cysteine Molecules. Cysteine ligand exchange 572 was performed using the previously reported method, ${ }^{14,33}$ with some 573 modifications. Briefly, $750 \mu \mathrm{L}$ of CdSe/CdS QDs in chloroform with 574 the concentration of $12 \mu \mathrm{M}$ was precipitated with methanol $(1 \mathrm{~mL}) .575$ Centrifugation was used to separate precipitated QDs from solution, 576 which were redissolved in chloroform $(750 \mu \mathrm{L})$. Then, $75 \mu \mathrm{L}$ of a 577 cysteine solution in methanol $(0.27 \mathrm{mM})$ was added to the QD 578 chloroform solution, shaken, and left for $2 \mathrm{~min}$. Next, $750 \mu \mathrm{L}$ of an 579 aqueous $0.01 \mathrm{M} \mathrm{KOH}$ solution was added, therefore adjusting the $\mathrm{pH} 580$ to 13 and forming a bilayer solution. The layers were then mixed by 581 gentle inversions multiple times until the majority of the QDs were 582 transferred to the aqueous layer, as indicated by color change. The 583 sample was then centrifuged in order to fully separate the layers and 584 remove aggregates (15 $000 \mathrm{rpm}, 1 \mathrm{~min}$ ). Finally, the aqueous layer 585 was extracted using a pipet and stored between 2 and $5{ }^{\circ} \mathrm{C}$. 586

Preparation of CdSe/CdS QD Solutions with Cys for NMR 587 Analysis. After ligand exchange, QDs still contained some amount of 588 residual hydrophobic molecules on the surface. In order to remove 589 those molecules, the QDs were purified after phase transfer by 590 washing with excess cysteine $(20 \mathrm{mg} / \mathrm{mL})$ using Millipore Sigma 591 
592 Amicon Ultra centrifugal filter units. To obtain a sample with a low 593 amount of Cys the excess cysteine was removed by washing of QDs 594 with a $\mathrm{pH} 13 \mathrm{KOH}$ solution. High Cys concentration solution $\mathrm{pH}$ was 595 adjusted to 13 via the addition of concentrated $\mathrm{KOH}$ solution. Cys596 QD solutions for NMR analysis were prepared and measured under 597 argon atmosphere to avoid Cys oxidation. $\mathrm{H}_{2} \mathrm{O}$ was used instead of $598 \mathrm{D}_{2} \mathrm{O}$ to avoid the replacement of $\mathrm{H}$ atoms in amino group of Cys with $599 \mathrm{D}$ atoms, which could influence the results of the measurements.

600 Preparation of CdSe/CdS QD Samples with Cys for FTIR 601 Analysis. After ligand exchange, QDs were washed several times with 602 an excess of Cys $(20 \mathrm{mg} / \mathrm{mL})$ to replace all the residual surfactant 603 ligands, which could be the cause of experimental artifacts using 604 Millipore Sigma Amicon Ultra centrifugal filter units. Sample with a 605 high amount of Cys were prepared via the precipitation of QDs with 606 acetone from the solution with a Cys concentration of $20 \mathrm{~g} / \mathrm{mL}$. To 607 obtain the sample with the low amount of Cys, the QDs were washed 608 two times with $\mathrm{KOH}$ solution with $\mathrm{pH} 13$ to gradually decrease the 609 amount of Cys and then were precipitated with acetone. Samples were 610 dried at a temperature of $70{ }^{\circ} \mathrm{C}$ overnight to remove remains of the 611 water and acetone.

612 Equipment. UV-vis absorption spectroscopy was performed using 613 a Cary 50 spectrophotometer (Varian, Australia). CD spectroscopy 614 was performed using a Jasco J-815 CD spectrometer operating under 615 a $\mathrm{N}_{2}$ flow of 5-8 L/min. TEM was performed using a FEI Titan 616 electron microscope operating at a beam voltage of $300 \mathrm{kV}$. FTIR 617 spectra were recorded on a Spectrum 100 instrument (PerkinElmer). 618 NMR studies were performed using a Bruker Avance III 400 NMR 619 spectrometer operating at $400.23 \mathrm{MHz}$ for ${ }^{1} \mathrm{H}$ and $100.64 \mathrm{MHz}$ for $620{ }^{13} \mathrm{C}$. The NMR spectra were acquired and processed using Bruker 621 Tospin 3.6 software. Standard ${ }^{1} \mathrm{H}$ and ${ }^{13} \mathrm{C}$ (proton -decoupled) pulse 622 sequences were taken from the Bruker pulse program library.

623 Computational Methods. DFT calculations reported in this 624 work were performed using projector-augmented wave (PAW) 625 pseudopotentials $^{70}$ and the Perdew-Burke-Ernzerhof (PBE) func626 tional, ${ }^{71}$ as implemented in the Vienna $A b$ Initio Simulation Package 627 (VASP) code, version 5.4.4. ${ }^{72,73}$ In order to determine the optimal 628 parameters for the optimization of the CdS bulk, the reciprocal space 629 was sampled using $\Gamma$-centered $k$-point grids of size $3 \times 3 \times 3,5 \times 5 \times$ $6305,7 \times 7 \times 7$, and $9 \times 9 \times 9$ and an energy cutoff of $500 \mathrm{eV}$. 631 Complying an energy convergence criterion of $1 \mathrm{meV} /$ atom, a $\Gamma$ 632 centered $k$-point grid of size $5 \times 5 \times 5$ was used for following bulk 633 calculations.

634 Periodic slab calculations were performed using a plane wave 635 kinetic energy cutoff of $500 \mathrm{eV}$ and a vacuum spacing of $15 \AA$ along 636 the $z$-axis, sampling the reciprocal space using a $\Gamma$-centered $k$-point 637 grid of size $3 \times 3 \times 1$, based on the optimized $k$-point density found in 638 the above bulk calculations. To model the surface of the core/shell 639 QD, a four-layer Cd-terminated CdS(0001) surface with a $(3 \times 3)$ 640 periodicity was employed for all the Cys adsorption calculations, 641 which is equivalent to the experimentally predominant (0002) plane 642 observed in the XRD patterns of $\mathrm{CdS}, \mathrm{CdSe}$, and $\mathrm{CdSe} / \mathrm{CdS}$ 643 heterostructures. $^{74}$ In these structures, atoms in the two topmost 644 layers were allowed to relax from their initial positions, whereas the 645 rest of the atoms were kept fixed at their bulk positions.

646 To assess the influence of Cys concentration on their binding 647 modes, an initial Cys molecule was adsorbed on the slab with a net 648 charge of -2 per molecule, which accounts for the 2 negative charges 649 from the deprotonated carboxyl and thiol groups in Cys at the 650 experimental $\mathrm{pH}$ ( $\mathrm{pH} 13)$. A total of 16 calculations were performed 651 for the adsorption of 1 Cys molecule on the surface, considering all 652 possible starting binding modes (Figure 3 ) while sampling all the 653 available surface adsorption sites. To simulate an increase in the 654 surface ligand concentration, the following calculations were 655 performed: (i) a second Cys was adsorbed on the lowest energy 656 structure containing 1 Cys ligand, and (ii) two Cys were adsorbed on 657 the pristine $\mathrm{CdS}(0001)$ surface to account for other potential binding 658 modes. This process was repeated with the addition of further Cys 659 molecules, until the computed $\Delta G_{\text {ads }}$ of an additional ligand became 660 endergonic. This procedure resulted in a total of 67 calculations; the 661 most stable ones are represented in Figure 8.
Gibbs corrections to the DFT-calculated potential energies were 662 computed for the QD surfaces with one adsorbed Cys at the 663 experimental temperature of $300 \mathrm{~K}$ and pressure of $1 \mathrm{~atm}$. These 664 corrections included the zero point energy (ZPE), vibrational 665 enthalpy, and entropy terms obtained by means of the harmonic 666 approximation using the Thermochemistry module implemented in 667 the Atomic Simulation Environment (ASE) package. ${ }^{75}$ The Gibbs 668 energy for the isolated Cys molecule was calculated at the same 669 temperature and pressure by means of the ideal gas approximation, 670 adding the ZPE and the translational, rotational, vibrational, and 671 electronic contributions of the constant-pressure heat capacity, plus a 672 $k_{\mathrm{B}}$ term. The Gibbs corrections obtained for the lowest energy 673 configurations with a single monodentate, bidentate, and tridentate 674 Cys ligand were employed to calculate the Gibbs corrections for 675 higher coverages. For instance, the Gibbs energy of a high coverage 676 model with 2 monodentate and 2 bidentate ligands was calculated as 677 follows:

$$
G_{2 \mathrm{mono}+2 \mathrm{bi}}=E_{2 \mathrm{mono}+2 \mathrm{bi}}+2 \Delta G_{\mathrm{mono}}+2 \Delta G_{\mathrm{bi}}
$$

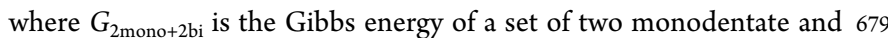
two bidentate Cys adsorbed on the surface, $E_{2 \text { mono+2bi }}$ is the potential 680 energy of the same system, and $\Delta G_{\text {mono }}$ and $\Delta G_{\mathrm{bi}}$ are the Gibbs 681 energy corrections of adsorbed monodentate and bidentate Cys 682 ligands on their own, in their lowest energy configurations. Gibbs 683 energies of adsorption of Cys molecules on the QD surface were 684 calculated with the following formula:

$$
\Delta G_{n \times \mathrm{Cys}^{*}}=G_{n \times \mathrm{Cys}^{*}}-n \times G_{\text {cys }}-E_{*}
$$

where $\Delta G_{n \times \mathrm{Cys}}^{*}$ is the Gibbs energy of adsorption of $n$ Cys molecules 686 (the asterisk symbol (*) indicates that the ligands are adsorbed on a 687 surface site), $G_{n \times \text { Cys }}^{*}$ is the total Gibbs energy of the system, $G_{\text {cys }}$ is the 688 Gibbs energy of a Cys molecule in the gas phase, and $E^{*}$ is the 689 potential energy of the clean surface.

690

Finally, noncovalent interactions within the ligand phase were 691 calculated by means of the Critic 2 software, ${ }^{76}$ by computing the 692 electron density, $\rho(r)$, and reduced density gradient, $s(r)$ :

$$
s(r)=\frac{|\nabla \rho(r)|}{2\left(3 \pi^{2}\right)^{1 / 3} \rho(r)^{4 / 3}}
$$

\section{ASSOCIATED CONTENT}

The Supporting Information is available free of charge on the 696 ACS Publications Web site at DOI: The Supporting 697 Information is available free of charge on the ACS Publications 698 website at DOI: 10.1021/acsnano.9b07513.

CdSe/CdS QD TEM and PL data, D-Cys CdSe/CdS 700 QD CD spectra, full free Cys, Cys-, and oleylamine- 701 capped CdSe/CdS QD FTIR spectra, differential 702 adsorption vs Cys coverage, NCI plots of DFT models. 703 All the DFT data underlying this work, including the 704 Cartesian coordinates of the modeled structures and 705 energies, are available at the following ioChem-BD 706 online dataset: 10.19061/iochem-bd-6-20 (PDF)

\section{AUTHOR INFORMATION}

\section{Corresponding Authors}

*E-mail: vkuznets@tcd.ie (V. A. Kuznetsova).

*E-mail: garciamm@tcd.ie (M. Garcia-Melchor).

*E-mail: igounko@tcd.ie (Y. K. Gun'ko).

Nadezda Prochukhan: 0000-0002-2535-7132

Finn Purcell-Milton: 0000-0002-3591-9477 
718 Yulia Gromova: 0000-0001-8826-8543

719 Max Garcia-Melchor: 0000-0003-1348-4692

720 Yurii K. Gun'ko: 0000-0002-4772-778X

\section{Author Contributions}

722 The manuscript was written through contributions of all 723 authors. All authors have given approval to the final version of 724 the manuscript.

\section{Author Contributions}

${ }_{726}{ }^{\nabla}$ These authors contributed equally.

727 Notes

728 The authors declare no competing financial interest.

\section{ACKNOWLEDGMENTS}

730 Authors gratefully thank Trinity College Dublin and SFI 731 Bioeconomy Research Centre, BEACON (No. SFI 16/RC/ 7323889 ) for financial support. Particularly, E.M.-T. acknowledges 733 funding from the Ussher Postgraduate Scholarships Pro734 gramme, generously funded through alumni donations and 735 Trinity's Commercial Revenue Unit. The DJEI/DES/SFI/ 736 HEA Irish Centre for High-End Computing (ICHEC) is also 737 acknowledged for the generous provision of computational 738 facilities and support. V.A.K. and Y.K.G. acknowledge support 739 from the Federal Target Program for Research and Develop740 ment of the Ministry of Science and Higher Education of the 741 Russian Federation (No. 14.587.21.0047, identifier RFME742 FI58718x0047).

\section{REFERENCES}

744 (1) Milton, F. P.; Govan, J.; Mukhina, M. V.; Gun'ko, Y. K. The 745 Chiral Nano-World: Chiroptically Active Quantum Nanostructures. 746 Nanoscale Horiz 2016, 1, 14-26.

747 (2) Ma, W.; Xu, L.; de Moura, A. F.; Wu, X.; Kuang, H.; Xu, C.; 748 Kotov, N. A. Chiral Inorganic Nanostructures. Chem. Rev. 2017, 117, 749 8041-8093.

750 (3) Moloney, M. P.; Gun'ko, Y. K.; Kelly, J. M. Chiral Highly 751 Luminescent CdS Quantum Dots. Chem. Commun. 2007, 38, 39007523902 .

753 (4) Ben-Moshe, A.; Maoz, B. M.; Govorov, A. O.; Markovich, G. 754 Chirality and Chiroptical Effects in Inorganic Nanocrystal Systems 755 with Plasmon and Exciton Resonances. Chem. Soc. Rev. 2013, 42, $7567028-7041$.

757 (5) Govan, J.; Gun'ko, Y. K. Recent Progress in Chiral Inorganic 758 Nanostructures. In Nanoscience, Vol. 3; O'Brien, P., Thomas, P. J., 759 Eds.; The Royal Society of Chemistry: London, 2016; pp 1-30.

760 (6) Kumar, J.; Thomas, K. G.; Liz-Marzán, L. M. Nanoscale 761 Chirality in Metal and Semiconductor Nanoparticles. Chem. Commun. 762 2016, 52, 12555-12569.

763 (7) Martynenko, I.; Kuznetsova, V.; Orlova, A.; Kanaev, P.; 764 Gromova, Y.; Maslov, V.; Baranov, A.; Fedorov, A. ZnSe/ZnS 765 Quantum Dots - Photosensitizer Complexes: Optical Properties and 766 Cancer Cell Photodynamic Destruction Effect. Proc. SPIE 2014, $76791263 \mathrm{C}$.

768 (8) Han, C.; Li, H. Chiral Recognition of Amino Acids Based on 769 Cyclodextrin-Capped Quantum Dots. Small 2008, 4, 1344-1350.

770 (9) Carrillo-Carrión, C.; Cárdenas, S.; Simonet, B. M.; Valcárcel, M. 771 Selective Quantification of Carnitine Enantiomers Using Chiral 772 Cysteine-Capped $\mathrm{CdSe}(\mathrm{ZnS})$ Quantum Dots. Anal. Chem. 2009, 773 81, 4730-4733.

774 (10) Xia, Y.; Zhou, Y.; Tang, Z. Chiral Inorganic Nanoparticles: 775 Origin, Optical Properties and Bioapplications. Nanoscale 2011, 3, 776 1374-1382.

777 (11) Li, Y.; Zhou, Y.; Wang, H.-Y.; Perrett, S.; Zhao, Y.; Tang, Z.; $778 \mathrm{Nie}, \mathrm{G}$. Chirality of Glutathione Surface Coating Affects the 779 Cytotoxicity of Quantum Dots. Angew. Chem., Int. Ed. 2011, 50, $7805860-5864$.
(12) Delgado-Pérez, T.; Bouchet, L. M.; de la Guardia, M.; Galian, 781 R. E.; Pérez-Prieto, J. Sensing Chiral Drugs by Using CdSe/ZnS 782 Nanoparticles Capped with N-Acetyl-L-Cysteine Methyl Ester. Chem. 783 - Eur. J. 2013, 19, 11068-11076.

(13) Ghasemi, F.; Hormozi-Nezhad, M. R.; Mahmoudi, M. Time- 785 Resolved Visual Chiral Discrimination of Cysteine Using Unmodified 786 CdTe Quantum Dots. Sci. Rep. 2017, 7, 890.

(14) Martynenko, I. V.; Kuznetsova, V. A.; Litvinov, I. K.; Orlova, A. 788 O.; Maslov, V. G.; Fedorov, A. V.; Dubavik, A.; Purcell-Milton, F.; 789 Gun'ko, Y. K.; Baranov, A. V. Enantioselective Cellular Uptake of 790 Chiral Semiconductor Nanocrystals. Nanotechnology 2016, 27, 791 075102-075109.

(15) Visheratina, A. K.; Purcell-Milton, F.; Serrano-García, R.; 793 Kuznetsova, V. A.; Orlova, A. O.; Fedorov, A. V.; Baranov, A. V.; 794 Gun'ko, Y. K. Chiral Recognition of Optically Active $\mathrm{CoFe}_{2} \mathrm{O}_{4} 795$ magnetic Nanoparticles by CdSe/CdS Quantum Dots Stabilised 796 with Chiral Ligands. J. Mater. Chem. C 2017, 5, 1692-1698. 797

(16) Kuznetsova, V. A.; Visheratina, A. K.; Ryan, A.; Martynenko, I. 798 V.; Loudon, A.; Maguire, C. M.; Purcell-Milton, F.; Orlova, A. O.; 799 Baranov, A. V.; Fedorov, A. V.; Prina-Mello, A.; Volkov, Y.; Gun'Ko, 800 Y. K. Enantioselective Cytotoxicity of ZnS:Mn Quantum Dots in 801 A549 Cells. Chirality 2017, 29, 403-408.

(17) Zhu, F.; Li, X.; Li, Y.; Yan, M.; Liu, S. Enantioselective Circular 803 Dichroism Sensing of Cysteine and Glutathione with Gold Nanorods. 804 Anal. Chem. 2015, 87, 357-361.

(18) Hou, X.; Ke, C.; Bruns, C. J.; McGonigal, P. R.; Pettman, R. B.; 806 Stoddart, J. F. Tunable Solid-State Fluorescent Materials for 807 Supramolecular Encryption. Nat. Commun. 2015, 6, 6884-6892. 808 (19) Li, W.-S.; Shen, Y.; Chen, Z.-J.; Cui, Q.; Li, S.-S.; Chen, L.-J. 809 Demonstration of Patterned Polymer-Stabilized Cholesteric Liquid 810 Crystal Textures for Anti-Counterfeiting Two-Dimensional Barcodes. 811 Appl. Opt. 2017, 56, 601-606.

812

(20) Zhu, Z.; Guo, J.; Liu, W.; Li, Z.; Han, B.; Zhang, W.; Tang, Z. 813 Controllable Optical Activity of Gold Nanorod and Chiral Quantum 814 Dot Assemblies. Angew. Chem., Int. Ed. 2013, 52, 13571-13575. 815

(21) Nguyen, T.-D.; Hamad, W. Y.; MacLachlan, M. J. CdS 816 Quantum Dots Encapsulated in Chiral Nematic Mesoporous Silica: 817 New Iridescent and Luminescent Materials. Adv. Funct. Mater. 2014, 818 24, 777-783.

819

(22) Hu, T.; Isaacoff, B. P.; Bahng, J. H.; Hao, C.; Zhou, Y.; Zhu, J.; 820 Li, X.; Wang, Z.; Liu, S.; Xu, C.; Biteen, J.; Kotov, N. A. Self- 821 Organization of Plasmonic and Excitonic Nanoparticles into Resonant 822 Chiral Supraparticle Assemblies. Nano Lett. 2014, 14, 6799-6810. 823

(23) Yeom, J.; Yeom, B.; Chan, H.; Smith, K. W.; Dominguez- 824 Medina, S.; Bahng, J. H.; Zhao, G.; Chang, W.-S.; Chang, S.-J.; 825 Chuvilin, A.; Melnikau, D.; Rogach, A. L.; Zhang, P.; Link, S.; Král, P.; 826 Kotov, N. A. Chiral Templating of Self-Assembling Nanostructures by 827 Circularly Polarized Light. Nat. Mater. 2015, 14, 66-72. 828

(24) Naaman, R.; Waldeck, D. H. Spintronics and Chirality: Spin 829 Selectivity in Electron Transport Through Chiral Molecules. Annu. 830 Rev. Phys. Chem. 2015, 66, 263-281.

831

(25) Bloom, B. P.; Kiran, V.; Varade, V.; Naaman, R.; Waldeck, D. 832 H. Spin Selective Charge Transport through Cysteine Capped CdSe 833 Quantum Dots. Nano Lett. 2016, 16, 4583-4589.

(26) Bloom, B. P.; Graff, B. M.; Ghosh, S.; Beratan, D. N.; Waldeck, 835 D. H. Chirality Control of Electron Transfer in Quantum Dot 836 Assemblies. J. Am. Chem. Soc. 2017, 139, 9038-9043. 837 (27) Varga, K.; Tannir, S.; Haynie, B. E.; Leonard, B. M.; Dzyuba, S. 838 V.; Kubelka, J.; Balaz, M. CdSe Quantum Dots Functionalized with 839 Chiral, Thiol-Free Carboxylic Acids: Unraveling Structural Require- 840 ments for Ligand-Induced Chirality. ACS Nano 2017, 11, 9846-9853. 841

(28) Choi, J. K.; Haynie, B. E.; Tohgha, U.; Pap, L.; Elliott, K. W.; 842 Leonard, B. M.; Dzyuba, S. V.; Varga, K.; Kubelka, J.; Balaz, M. 843 Chirality Inversion of $\mathrm{CdSe}$ and $\mathrm{CdS}$ Quantum Dots without 844 Changing the Stereochemistry of the Capping Ligand. ACS Nano 845 2016, 10, 3809-3815.

(29) Tohgha, U.; Deol, K. K.; Porter, A. G.; Bartko, S. G.; Choi, J. 847 K.; Leonard, B. M.; Varga, K.; Kubelka, J.; Muller, G.; Balaz, M. 848 Ligand Induced Circular Dichroism and Circularly Polarized 849 
850 Luminescence in CdSe Quantum Dots. ACS Nano 2013, 7, 1109485111102.

852 (30) Tohgha, U.; Varga, K.; Balaz, M. Achiral CdSe Quantum Dots 853 Exhibit Optical Activity in the Visible Region upon Post-Synthetic 854 Ligand Exchange with d- or l-Cysteine. Chem. Commun. 2013, 49, 855 1844-1846.

856 (31) Cheng, J.; Hao, J.; Liu, H.; Li, J.; Li, J.; Zhu, X.; Lin, X.; Wang, 857 K.; He, T. Optically Active CdSe-Dot/CdS-Rod Nanocrystals with 858 Induced Chirality and Circularly Polarized Luminescence. ACS Nano 859 2018, 12, 5341-5350.

860 (32) Puri, M.; Ferry, V. E. Circular Dichroism of CdSe Nanocrystals 861 Bound by Chiral Carboxylic Acids. ACS Nano 2017, 11, 1224086212246.

863 (33) Purcell-Milton, F.; Visheratina, A. K.; Kuznetsova, V. A.; Ryan, 864 A.; Orlova, A. O.; Gun'ko, Y. K. Impact of Shell Thickness on 865 Photoluminescence and Optical Activity in Chiral CdSe/CdS Core/ 866 Shell Quantum Dots. ACS Nano 2017, 11, 9207-9214.

867 (34) Martynenko, I. V.; Baimuratov, A. S.; Osipova, V. A.; 868 Kuznetsova, V. A.; Purcell-Milton, F.; Rukhlenko, I. D.; Fedorov, A. 869 V.; Gun'Ko, Y. K.; Resch-Genger, U.; Baranov, A. V. Excitation 870 Energy Dependence of the Photoluminescence Quantum Yield of 871 Core/Shell CdSe/CdS Quantum Dots and Correlation with Circular 872 Dichroism. Chem. Mater. 2018, 30, 465-471.

873 (35) Moloney, M. P.; Govan, J.; Loudon, A.; Mukhina, M.; Gun'ko, 874 Y. K. Preparation of Chiral Quantum Dots. Nat. Protoc. 2015, 10, 875 558-573.

876 (36) Mukhina, M. V.; Maslov, V. G.; Baranov, A. V.; Fedorov, A. V.; 877 Orlova, A. O.; Purcell-Milton, F.; Govan, J.; Gun'ko, Y. K. Intrinsic 878 Chirality of CdSe/ZnS Quantum Dots and Quantum Rods. Nano 879 Lett. 2015, 15, 2844-2851.

880 (37) Mukhina, M. V.; Maslov, V. G.; Korsakov, I. V.; Purcell Milton, 881 F.; Loudon, A.; Baranov, A. V.; Fedorov, A. V.; Gun'ko, Y. K. 882 Optically Active II-VI Semiconductor Nanocrystals via Chiral Phase 883 Transfer. MRS Proc. 2015, 1793, 27-33.

884 (38) Ben Moshe, A.; Szwarcman, D.; Markovich, G. Size 885 Dependence of Chiroptical Activity in Colloidal Quantum Dots. 886 ACS Nano 2011, 5, 9034-9043.

887 (39) Ben Moshe, A.; Markovich, G.; Tang, Z. Optically Active and 888 Chiral Semiconductor Nanocrystals. In Chiral Nanomaterials: 889 Preparation, Properties and Applications; Tang, Z., Ed.; Wiley-VCH: 890 Weinheim, Germany; 2017; pp 85-98.

891 (40) Ben-Moshe, A.; Teitelboim, A.; Oron, D.; Markovich, G. 892 Probing the Interaction of Quantum Dots with Chiral Capping 893 Molecules Using Circular Dichroism Spectroscopy. Nano Lett. 2016, 894 16, 7467-7473.

895 (41) Elliott, S. D.; Moloney, M. P.; Gun'ko, Y. K. Chiral Shells and 896 Achiral Cores in CdS Quantum Dots. Nano Lett. 2008, 8, 2452-2457. 897 (42) Zhou, Y.; Yang, M.; Sun, K.; Tang, Z.; Kotov, N. A. Similar 898 Topological Origin of Chiral Centers in Organic and Nanoscale 899 Inorganic Structures: Effect of Stabilizer Chirality on Optical 900 Isomerism and Growth of CdTe Nanocrystals. J. Am. Chem. Soc. 901 2010, 132, 6006-6013.

902 (43) Zhou, Y.; Zhu, Z.; Huang, W.; Liu, W.; Wu, S.; Liu, X.; Gao, Y.; 903 Zhang, W.; Tang, Z. Optical Coupling Between Chiral Biomolecules 904 and Semiconductor Nanoparticles: Size-Dependent Circular Dichro905 ism Absorption. Angew. Chem., Int. Ed. 2011, 50, 11456-11459.

906 (44) Govorov, A. O.; Fan, Z.; Hernandez, P.; Slocik, J. M.; Naik, R. 907 R. Theory of Circular Dichroism of Nanomaterials Comprising Chiral 908 Molecules and Nanocrystals: Plasmon Enhancement, Dipole Inter909 actions, and Dielectric Effects. Nano Lett. 2010, 10, 1374-1382.

910 (45) Tsay, J. M.; Doose, S.; Pinaud, F.; Weiss, S. Enhancing the 911 Photoluminescence of Peptide-Coated Nanocrystals with Shell 912 Composition and UV Irradiation. J. Phys. Chem. B 2005, 109, 913 1669-1674.

914 (46) Harris, R. D.; Amin, V. A.; Lau, B.; Weiss, E. A. Role of 915 Interligand Coupling in Determining the Interfacial Electronic 916 Structure of Colloidal CdS Quantum Dots. ACS Nano 2016, 10, 917 1395-1403.
(47) Visheratina, A. K.; Orlova, A. O.; Purcell-Milton, F.; 918 Kuznetsova, V. A.; Visheratin, A. A.; Kundelev, E. V.; Maslov, V. 919 G.; Baranov, A. V.; Fedorov, A. V.; Gun'Ko, Y. K. Influence of CdSe 920 and CdSe/CdS Nanocrystals on the Optical Activity of Chiral 921 Organic Molecules. J. Mater. Chem. C 2018, 6, 1759-1766.

(48) Mukhina, M. V.; Maslov, V. G.; Baranov, A. V.; Artemyev, M. 923 V.; Orlova, A. O.; Fedorov, A. V. Anisotropy of Optical Transitions in 924 Ordered Ensemble of CdSe Quantum Rods. Opt. Lett. 2013, 38, 925 $3426-3428$.

926

(49) Gao, X.; Zhang, X.; Deng, K.; Han, B.; Zhao, L.; Wu, M.; Shi, 927 L.; Lv, J.; Tang, Z. Excitonic Circular Dichroism of Chiral Quantum 928 Rods. J. Am. Chem. Soc. 2017, 139, 8734-8739.

(50) Govan, J. E.; Jan, E.; Querejeta, A.; Kotov, N. A.; Gun'ko, Y. K. 930 Chiral Luminescent CdS Nano-Tetrapods. Chem. Commun. 2010, 46, 931 6072-6074.

(51) Frederick, M. T.; Amin, V. A.; Swenson, N. K.; Ho, A. Y.; 933 Weiss, E. A. Control of Exciton Confinement in Quantum Dot- 934 Organic Complexes through Energetic Alignment of Interfacial 935 Orbitals. Nano Lett. 2013, 13, 287-292.

936

(52) Li, T.; Park, H. G.; Lee, H.-S.; Choi, S.-H. Circular Dichroism 937 Study of Chiral Biomolecules Conjugated with Silver Nanoparticles. 938 Nanotechnology 2004, 15, S660-S663.

(53) Mahler, B.; Spinicelli, P.; Buil, S.; Quelin, X.; Hermier, J.-P.; 940 Dubertret, B. Towards Non-Blinking Colloidal Quantum Dots. Nat. 941 Mater. 2008, 7, 659.

(54) Gautier, C.; Bürgi, T. Chiral Inversion of Gold Nanoparticles. J. 943 Am. Chem. Soc. 2008, 130, 7077-7084.

(55) Kuno, J; Imamura, Y; Katouda, M. Tashiro, M.; Kawai, T, 945 Nakashima, T. Inversion of Optical Activity in the Synthesis of 946 Mercury Sulfide Nanoparticles: Role of Ligand Coordination. Angew. 947 Chem., Int. Ed. 2018, 57, 12022-12026.

(56) Kurihara, T.; Matano, A.; Noda, Y.; Takegoshi, K. Rotational 949 Motion of Ligand-Cysteine on CdSe Magic-Sized Clusters. J. Phys. 950 Chem. C 2019, 123, 14993-14998.

(57) Kurihara, T.; Noda, Y.; Takegoshi, K. Capping Structure of 952 Ligand-Cysteine on CdSe Magic-Sized Clusters. ACS Omega 2019, 953 4, 3476-3483.

(58) Kurihara, T.; Noda, Y.; Takegoshi, K. Quantitative Solid-State 955 NMR Study on Ligand-Surface Interaction in Cysteine-Capped 956 CdSe Magic-Sized Clusters. J. Phys. Chem. Lett. 2017, 8, 2555-2559. 957

(59) Cui, Y.; Lou, Z.; Wang, X.; Yu, S.; Yang, M. A Study of Optical 958 Absorption of Cysteine-Capped CdSe Nanoclusters Using First- 959 Principles Calculations. Phys. Chem. Chem. Phys. 2015, 17, 9222- 960 9230.

961

(60) Mukhina, M. V.; Korsakov, I. V.; Maslov, V. G.; Purcell-Milton, 962 F.; Govan, J.; Baranov, A. V.; Fedorov, A. V.; Gun'ko, Y. K. Molecular 963 Recognition of Biomolecules by Chiral CdSe Quantum Dots. Sci. Rep. 964 2016, 6, 24177.

965

(61) Kane-Maguire, L. A. P.; Riley, P. J. High Field NMR Study of 966 the Binding of Lead(II) to Cysteine and Glutathione. J. Coord. Chem. 967 1993, 28, 105-120.

(62) Drauz, K.; Grayson, I.; Kleemann, A.; Krimmer, H.-P.; 969 Leuchtenberger, W.; Weckbecker, C. Amino Acids. In Ullmann's 970 Encyclopedia of Industrial Chemistry; Wiley-VCH Verlag GmbH \& 971 Co. KGaA, 2007.

(63) Friedman, J. I.; McMahon, M. T.; Stivers, J. T.; Van Zijl, P. C. 973 M. Indirect Detection of Labile Solute Proton Spectra via the Water 974 Signal Using Frequency-Labeled Exchange (FLEX) Transfer. J. Am. 975 Chem. Soc. 2010, 132, 1813-1815.

976

(64) Zong, X.; Wang, P.; Kim, S.-G.; Jin, T. Sensitivity and Source of 977 Amine-Proton Exchange and Amide-Proton Transfer Magnetic 978 Resonance Imaging in Cerebral Ischemia. Magn. Reson. Med. 2014, 979 $71,118-132$. 980

(65) Cistola, D.; M Small, D.; Hamilton, J. Ionization Behavior of 981 Aqueous Short-Chain Carboxylic Acids: A Carbon- ${ }^{13}$ NMR Study. J. 982 Lipid Res. 1982, 23, 795-799.

(66) Abraham, A.; Mihaliuk, E.; Kumar, B.; Legleiter, J.; Gullion, T. 984 Solid-State NMR Study of Cysteine on Gold Nanoparticles. J. Phys. 985 Chem. C 2010, 114, 18109-18114. 
987 (67) Akbarzadeh, M.; Moosavi-Movahedi, Z.; Shockravi, A.; Jafari, 988 R.; Nazari, K.; Sheibani, N.; Moosavi-Movahedi, A. A. Metallo989 Vesicular Catalysis: A Mixture of Vesicular Cysteine/Iron Mediates 990 Oxidative pH Switchable Catalysis. J. Mol. Catal. A: Chem. 2016, 424, 991 181-193.

992 (68) Contreras-García, J.; Boto, R.; Izquierdo-Ruiz, F.; Reva, I.; 993 Woller, T.; Alonso, M. A Benchmark for the Non-Covalent 994 Interaction (NCI) Index Or... Is It Really All in the Geometry? 995 Theor. Chem. Acc. 2016, 135, DOI: 10.1007/s00214-016-1977-7.

996 (69) Johnson, E. R.; Keinan, S.; Mori-Sánchez, P.; Contreras-García, 997 J.; Cohen, A. J.; Yang, W. Revealing Noncovalent Interactions. J. Am. 998 Chem. Soc. 2010, 132, 6498-6506.

999 (70) Blöchl, P. E. Projector Augmented-Wave Method. Phys. Rev. B: 1000 Condens. Matter Mater. Phys. 1994, 50, 17953-17979.

1001 (71) Perdew, J. P.; Burke, K.; Ernzerhof, M. Generalized Gradient 1002 Approximation Made Simple. Phys. Rev. Lett. 1996, 77, 3865-3868. 1003 (72) Kresse, G.; Furthmüller, J. Efficiency of Ab Initio Total Energy 1004 Calculations for Metals and Semiconductors Using a Plane-Wave 1005 Basis Set. Comput. Mater. Sci. 1996, 6, 15-50.

1006 (73) Kresse, G.; Furthmüller, J. Efficient Iterative Schemes for $A b$ 1007 Initio Total-Energy Calculations Using a Plane-Wave Basis Set. Phys. 1008 Rev. B: Condens. Matter Mater. Phys. 1996, 54, 11169-11186.

1009 (74) Chauhan, H.; Kumar, Y.; Deka, S. New Synthesis of Two1010 Dimensional CdSe/CdS Core@shell Dot-in-Hexagonal Platelet 1011 Nanoheterostructures with Interesting Optical Properties. Nanoscale 1012 2014, 6, 10347-10354.

1013 (75) Hjorth Larsen, A.; Jørgen Mortensen, J.; Blomqvist, J.; Castelli, 1014 I. E.; Christensen, R.; Dułak, M.; Friis, J.; Groves, M. N.; Hammer, B.; 1015 Hargus, C.; Hermes, E. D.; Jennings, P. C.; Bjerre Jensen, P.; 1016 Kermode, J.; Kitchin, J. R.; Leonhard Kolsbjerg, E.; Kubal, J.; 1017 Kaasbjerg, K.; Lysgaard, S.; Bergmann Maronsson, J.; et al. The 1018 Atomic Simulation Environment-a Python Library for Working with 1019 Atoms. J. Phys.: Condens. Matter 2017, 29, 273002-273033.

1020 (76) Otero-de-la-Roza, A.; Johnson, E. R.; Luaña, V. Critic2: A 1021 Program for Real-Space Analysis of Quantum Chemical Interactions 1022 in Solids. Comput. Phys. Commun. 2014, 185, 1007-1018. 